\title{
Evolution of the discrepancy between a universe and its model
}

\author{
MAsafumi Seriu \\ Institute of Cosmology \\ Department of Physics \& Astronomy \\ Tufts University \\ Massachusetts 02155, USA \\ $*$ \\ Department of Physics, Fukui University \\ Fukui 910-8507, Japan日
}

\begin{abstract}
We study a fundamental issue in cosmology: Whether we can rely on a cosmological model to understand the real history of the Universe. This fundamental, still unresolved issue is often called the "model-fitting problem (or averaging problem) in cosmology". Here we analyze this issue with the help of the spectral scheme prepared in the preceding studies.

Choosing two specific spatial geometries $\mathcal{G}$ and $\mathcal{G}^{\prime}$ that are very close to each other, we investigate explicitly the time evolution of the spectral distance between them; as two spatial geometries $\mathcal{G}$ and $\mathcal{G}^{\prime}$, we choose a flat 3 -torus and a perturbed geometry around it, mimicking the relation of a "model universe" and the "real Universe". Then we estimate the spectral distance between them, $d_{N}\left(\mathcal{G}, \mathcal{G}^{\prime}\right)$, and investigate its time evolution explicitly. This analysis is done efficiently by making use of the basic results of the standard linear structure-formation theory.

We observe that, as far as the linear approximation of the geometrical perturbation is valid, $d_{N}\left(\mathcal{G}, \mathcal{G}^{\prime}\right)$ does not increase with time prominently, rather it shows the tendency to decrease. This result is compatible with the general belief in the reliability of describing the Universe by means of a model, and calls for more detailed studies along the same line including the investigation of wider class of spacetimes and the analysis beyond the linear regime.
\end{abstract}

\section{Introduction}

We study in this paper one of the fundamental issues in cosmology: How precisely a cosmological model traces the time evolution of the real Universe. This significant problem has been raised and studied for a long time but we have not

\footnotetext{
${ }^{1}$ Present address. E-mail: mseriu@edu00.f-edu.fukui-u.ac.jp
} 
yet caught a glimpse of a satisfactory understanding of this issue. It is often called the "model-fitting problem in cosmology" or the "averaging problem in cosmology" [1, 2].

This problem originates from the very nature of cosmology. Cosmology is our attempt to grasp our Universe by reference to a model. The real Universe is full of complexity while a model, which serves as a format of perceiving reality, is much simpler than the reality itself. In cosmology, thus, there is in principle an inevitable discrepancy between the reality and its model.

When we try to guess the past and future of the Universe based on its model, thus, we need a guarantee that the inevitable discrepancy between the reality and its model does not develop so much within the time scale of concern. Considering the highly nonlinear nature of the Einstein equation, the enlargement of the discrepancy would not be a very surprising result even if it were the case. Therefore this issue should be thoroughly clarified before we state anything meaningful for the past or future of our Universe. One might symbolically state the problem depicted here as "Is cosmology possible?"

The central difficulty of dealing with the issue of inevitable discrepancy may arise from the following two facts:

(D1) It is difficult to define a reasonable procedure of "averaging geometry" in a self-consistent manner. For instance, it is difficult to define the averaged spatial metric $\left\langle h_{a b}\right\rangle$ in a spatial-diffeo-invariant manner.

(D2) On one hand, we need to handle two or more universes (viz. the real one and its models) at the same time; on the other hand, there has been no efficient mathematical language for comparing two or more geometries with each other.

At first sight, the difficulties (D1) and (D2) look mutually independent. When we contemplate on them deeper, however, we realize that they are both rooted in the issue of how to quantify an intuitive statement "the two shapes look similar": The issue (D1) is reduced to the issue of how to relate the averaged geometry to the original geometry in a reasonable manner. The issue (D2) is about the comparison procedure for two given geometries. Extracting the essence from (D1) and (D2), then, we infer that the concept of "closeness" between two geometries would play a key role. We pursue this viewpoint further in this paper, making use of our preceding related studies.

In preceding work we have indeed prepared a framework in which the measure of closeness between geometries plays a central role [3, 4, 5], and have made some preliminary investigations on the model-fitting problem based on this framework [6, 7]. (For simplicity we call the framework spectral scheme hereafter). The purpose of this paper is to make a more explicit investigation by the spectral scheme on the time evolution of the discrepancy between two nearby geometries, $\mathcal{G}$ and $\mathcal{G}^{\prime}$, by choosing concrete, tractable geometries for $\mathcal{G}$ and $\mathcal{G}^{\prime}$. 
Before going into details, let us first discuss why handling the discrepancy between two universes is so difficult and how we try to tackle this problem by introducing the spectral scheme.

For this purpose the following example may clearly illustrate the situation. Let $\mathcal{G}_{0}$ be some topologically complicated space, with various small topological handles attached [8, 3]. When we observe $\mathcal{G}_{0}$ at the energy scale $E$, the handles smaller than $E^{-1}$ would not be observed, resulting in a simpler effective geometry, say $\mathcal{G}_{E}$. If the energy scale is decreased further to $E^{\prime}\left(E>E^{\prime}\right)$, the handles smaller than $E^{\prime-1}$ effectively disappear, resulting in a much simpler effective geometry $\mathcal{G}_{E^{\prime}}$. A similar phenomenon occurs when we change observational apparatus, instead of changing $E$; a portion of geometrical information incompatible with the observational apparatus would in effect disappear so that the resulting effective geometry depends on the apparatus used.

In this way, we are led to the concept of effective geometry, viz. geometrical structure varying as a "function" of observational energy $E$ and observational apparatus $A$, which may be indicated by $\mathcal{G}_{(E, A)}$. We intuitively know that $\mathcal{G}_{(E, A)}$ is effectively "close" to $\mathcal{G}_{0}$ and that $\mathcal{G}_{(E, A)}$ can serve as a model for $\mathcal{G}_{0}$ when we are interested in the phenomena labeled by $(E, A)$.

On the other hand, $\mathcal{G}_{(E, A)}$ (for some $E$ and $A$ ) is totally different from the original geometry $\mathcal{G}_{0}$ from the viewpoint of traditional mathematical theories of geometry and topology. Even though $\mathcal{G}_{(E, A)}$ and $\mathcal{G}_{0}$ are physically "close" to each other, mathematics classifies them into different topology classes. Topology is a scale-free concept and the scale of a handle is not counted, while in spacetime physics it should also be taken into account because the observational energy scale enters into the argument $[8,3]$.

The central difficulty of analyzing the issue of discrepancy is, then, that we are lacking in a scheme of geometrical approximation which makes it possible to regard $\mathcal{G}_{(E, A)}$ as an approximation (a "model") of $\mathcal{G}_{0}$, even though they are totally different in a conservative sense. Thus the first thing to do is to construct a new framework which justifies our intuition that $\mathcal{G}_{(E, A)}$ is "close" to $\mathcal{G}_{0}$ in a certain sense. In this way we return to the issue of "closeness" discussed after (D1) and (D2).

With the above consideration, it is now clear that the following points should be clarified;

(a) How to quantify "discrepancy" or "closeness" between two geometries.

(b) How to take into account the "coarseness" of truncating information of geometry. (Scale-dependence of effective geometry.)

(c) How to take into account the type of apparatus used for collecting information of geometry. (Apparatus-dependence of effective geometry.)

It has been shown that a measure of closeness between two geometries, say $\mathcal{G}$ and $\mathcal{G}^{\prime}$, can be defined in terms of the spectra [3]; here the term "spectra" means 
a sequence of eigenvalues of a certain elliptic Hermitian operator, numbered in an increasing order. (We mainly consider the Laplacian $\Delta$ as a typical Hermitian elliptic operator though the basic idea itself is universal.) The measure of closeness is defined by comparing the spectra for $\mathcal{G}$ with those for $\mathcal{G}^{\prime}$ (Issue $(a)$ ). Furthermore, only the first $N$ spectra $\left(\lambda_{1}, \lambda_{2}, \cdots, \lambda_{N}\right)$ are compared, and in this manner, a natural cut-off scale $\lambda_{N}^{-1 / 2}$ is purposefully introduced. This procedure can be symbolically described as "comparing the sound of $\mathcal{G}$ with the sound of $\mathcal{G}^{\prime} "$.

First of all, the spectra are the spatial-diffeomorphism invariant so that they are desirable quantities for quantifying "closeness" (see (D1)). Next, on dimensional grounds, the lower (higher) spectra reflect the larger (smaller) scale properties of the geometry. By comparing only the low-lying spectra up to the $N$-th spectrum $\lambda_{N}$, thus, $\mathcal{G}$ and $\mathcal{G}^{\prime}$ are compared in a coarse-grained manner, neglecting the difference smaller than the cut-off scale corresponding to $\lambda_{N}$. Thus the measure of closeness $d_{N}\left(\mathcal{G}, \mathcal{G}^{\prime}\right)$ introduced in this way naturally takes care of the scale-dependent nature of spatial geometry (Issue (b)). Finally, the choice of the Hermitian operator determines the type of vibration modes used for probing geometry, so that in principle it corresponds to the observational apparatus used for measuring geometry. Thus the choice of the operator defines which aspects of geometry are compared to measure the closeness between the spaces. As a result, our measure of closeness becomes apparatus-dependent, too (Issue (c)).

In this manner, we are now capable of quantifying the "closeness" between two given geometries as a "function" of the coarse-graining scale and the observational apparatus [3]. For a given geometry $\mathcal{G}^{\prime}$, the optimal model of $\mathcal{G}^{\prime}$ among a set of models can be defined as the model geometry $\mathcal{G}$ closest to $\mathcal{G}^{\prime}$ when measured by $d_{N}$. Therefore we realize that a suitable model for a given "reality" $\mathcal{G}^{\prime}$ is not unique, but it varies in accordance with the observational scale and apparatus [6, 7]. The mapping $\mathcal{G}^{\prime} \mapsto \mathcal{G}$ here may be interpreted as "averaging the geometry $\mathcal{G}^{\prime}$ " if one wishes. The unique feature of this approach is, however, it does not resort to the ambiguous averaging procedures such as defining the "averaged metric" $\left\langle h_{a b}\right\rangle$; rather the whole of the geometry is directly mapped to some suitable model geometry.

Furthermore, it has been shown that the space of all spaces $\mathcal{S}_{N}$, which is a set of all compact Riemannian geometries equipped with the measure of closeness $d_{N}$, forms a metrizable space [4]. This result makes it justified to call the measure of closeness $d_{N}\left(\mathcal{G}, \mathcal{G}^{\prime}\right)$ as the spectral distance between $\mathcal{G}$ and $\mathcal{G}^{\prime}$. At the same time, it means that we have constructed a basic arena $\mathcal{S}_{N}$ for analyzing fundamental problems in spacetime physics, such as the one discussed in the present paper.

One of the advantages of the spectral scheme is that the basic quantities $\left\{\lambda_{n}\right\}$ carry clear, well-defined meaning both physically and mathematically. Physically they represent the vibration modes of the space when "tapped" by a certain apparatus $(\Delta)$; mathematically they are the eigenvalues of a certain elliptic operator 
defined on a space and their investigation forms one active subject in geometry.

In reality, it is true that only a restricted number of cases are known for which whole of the spectra, $\left\{\lambda_{n}\right\}_{n=1}^{\infty}$, can be obtained explicitly. Even so, it is still meaningful to construct a general framework based on $\left\{\lambda_{n}\right\}$ : First, let us recall that we do not need the whole of the spectra but only the first $N$ spectra $\left(\lambda_{1}, \lambda_{2}, \cdots, \lambda_{N}\right)$ to be compared. It is known that the lower spectra are relatively easier to obtain by, for instance, the numerical calculations 8 as well as analytical methods [10], and these lower spectra contain enough information on the global nature of the space. Second, in many cases, we are more interested in the difference of the spectra $\left(\delta \lambda_{n}\right)$ between two geometries (or between two neighboring time-slices in one spacetime) than the spectra themselves, and usually we can estimate the former even when the latter are not explicitly available. Third, it is meaningful that the spectra are theoretically well-defined and in principle determined, even though not determined explicitly in many cases. P Finally, the situation is not proper to the spectral scheme; whatever we choose as a framework of analysis, investigations of complicated geometries do not go straightforwardly anyway.

It is now possible to picture the situation in question as follows: Each point in the space $\mathcal{S}_{N}$ indicates a certain geometry viewed with the apparatus $\Delta$ at the scale $\lambda_{N}$. . A set of models is represented by the points distributed throughout $\mathcal{S}_{N}$. The optimal model $\mathcal{G}$ is then represented by the point among them closest to the point representing the reality $\mathcal{G}^{\prime}$ [6]. Thus, the real geometry $\mathcal{G}^{\prime}$ and its model geometry $\mathcal{G}$ are represented by two points that are very close to each other in $\mathcal{S}_{N}$. These two points move in time and follow two trajectories in $\mathcal{S}_{N}$, and the relative behavior of these two trajectories is crucial for a model $\mathcal{G}$ to be a good approximation of $\mathcal{G}^{\prime}$. What is essential is, then, to investigate the time evolution of the spectral distance $d_{N}\left(\mathcal{G}, \mathcal{G}^{\prime}\right)$. For this purpose, the evolution equations for the spectra of the universe is required since the spectral distance is defined solely in terms of the spectra. The spectral evolution equations have been indeed derived[5], and they can be regarded as the spectral representation of the Einstein equation. Now we have constructed a formalism which consists of the following triad; (I) the spectral distance $d_{N}$, (II) the space of all spaces $\mathcal{S}_{N}$ and (III) the spectral evolution equations.

In this paper, we apply the spectral scheme explicitly to a concrete, tractable situation to get deeper understanding of the relation between the reality and its

\footnotetext{
${ }^{2}$ Indeed there are already several numerical calculations [9] (based on the finite element method) which provide the first 15 spectra or so for the case of 3-dimensional hyperbolic geometries with non-trivial topologies. This kind of numerical techniques are certain to develop in the near future.

${ }^{3}$ The situation here is very similar to the one for quantum field theory on a curved spacetime. There are only restricted number of spacetimes available for which we can explicitly obtain the complete set of positive frequency solutions of the field equation. However the general framework of quantum field theory is still worth constructing.

${ }^{4}$ More rigorously, it indicates a class of geometries that look similar to each other when viewed with the apparatus $\Delta$ at the scale $\lambda_{N}$.
} 
model. We construct a tractable pair of spaces imitating the relation between the real Universe and its model, and estimate the time evolution of their spectral distance explicitly. As the first investigation in this direction, we here investigate only the linear-regime, viz. the period when the discrepancy can be regarded as small. Though the situation analyzed here is inevitably limited, the present analysis would give us insight for the cases when the discrepancy is larger than the present case and would provide a motivation for further studies for these cases.

In Section 2, we will summarize the basic formulas in the spectral scheme only to a necessary extent for the analysis in this paper. In Section 3, we construct two spacetimes, mimicking the relation between the Universe and its model; as for their spatial sections, a flat 3-torus is chosen for what is regard as a "model universe" $(\mathcal{G})$, while a perturbed geometry around $\mathcal{G}$ is chosen for what plays the role of the "real Universe" $\left(\mathcal{G}^{\prime}\right)$. Then we prepare several fundamental quantities and formulas for these spacetimes in terms of the spectral scheme. We combine the standard linear-perturbation theory with the spectral scheme in Section $⿴$, and derive the time-dependence of various coefficients necessary for our analysis. With these preparations, we estimate the time-development of the spectral distance between $\mathcal{G}$ and $\mathcal{G}^{\prime}$ in Section 5 (the main results are Eqs.46)-(49)). Section 6 is devoted for several discussions.

\section{Fundamental formulas in the spectral scheme}

Let us recall some results in the spectral scheme necessary for later analysis. If

Let us consider a geometry $\mathcal{G}$ described by a $(D-1)$-dimensional compact Riemannian manifold without boundaries, $(\Sigma, h)$.

We consider an eigenvalue problem of the Laplacian $\Delta$ on $(\Sigma, h), \Delta f=-\lambda f$. Then we get the spectra, viz. the sequence of eigenvalues arranged in an increasing order, $\left\{\lambda_{n}\right\}_{n=0,1,2, \cdots}:=\left\{0=\lambda_{0}<\lambda_{1} \leq \lambda_{2} \leq \cdots \leq \lambda_{n} \leq \cdots\right\}$. At the same time, we get the set of real-valued eigenfunctions corresponding to the spectra, $\left\{f_{n}\right\}_{n=0,1,2, \ldots}$, normalized as $\left(f_{m}, f_{n}\right):=\int_{\Sigma} f_{m} f_{n} \sqrt{ }=\delta_{m n}$, where the natural integral measure on $(\Sigma, h)$ is indicated by $\sqrt{ }:=\sqrt{\operatorname{det}\left(h_{a b}\right)}$.

Now we consider two geometries $\mathcal{G}$ and $\mathcal{G}^{\prime}$. Let $\left\{\lambda_{m}\right\}_{m=0}^{\infty}$ and $\left\{\lambda_{n}^{\prime}\right\}_{n=0}^{\infty}$ be the spectra for $\mathcal{G}$ and for $\mathcal{G}^{\prime}$, respectively. Then the spectral measure of closeness between $\mathcal{G}$ and $\mathcal{G}^{\prime}$ of order $N, d_{N}\left(\mathcal{G}, \mathcal{G}^{\prime}\right)$, is defined as $\llbracket$, 6]

$$
d_{N}\left(\mathcal{G}, \mathcal{G}^{\prime}\right):=\sum_{n=1}^{N} \mathcal{F}\left(\frac{\lambda_{n}^{\prime}}{\lambda_{n}}\right),
$$

\footnotetext{
${ }^{5}$ The reader is advised to refer to Refs. [3], [4] and [5] for more details.

${ }^{6}$ In this section, $D$ indicates the dimension of a spacetime, so that its spatial section becomes $(D-1)$-dimensional.
} 
where $\mathcal{F}(x)(x>0)$ is any continuous function satisfying

$$
\begin{aligned}
\mathcal{F}(1) & =0, \\
\mathcal{F}(y) & >\mathcal{F}(x) \text { for } y>x \geq 1, \\
\mathcal{F}(1 / x) & =\mathcal{F}(x) .
\end{aligned}
$$

For practical applications, it is convenient to assume further $(i) \mathcal{F}(x)$ is smooth at $x=1$ (then $\mathcal{F}^{\prime}(1)=0$ and $\mathcal{F}^{\prime \prime}(1) \geq 0$ from the conditions (2)) and (ii) $\mathcal{F}^{\prime \prime}(1)>0$. The postulation $(i i)$ is for making $d_{N}\left(\mathcal{G}, \mathcal{G}^{\prime}\right)$ sensitive enough to detect a fine difference between $\mathcal{G}$ and $\mathcal{G}^{\prime}$ when they are very close to each other (see Eq. (10)).

It is convenient to set $\mathcal{F}$ to be $\mathcal{F}_{1}(x)=\frac{1}{2} \ln \frac{1}{2}(\sqrt{x}+1 / \sqrt{x})$. Then Eq.(1) becomes [3]

$$
d_{N}\left(\mathcal{G}, \mathcal{G}^{\prime}\right)=\frac{1}{2} \sum_{n=1}^{N} \ln \frac{1}{2}\left(\sqrt{\frac{\lambda_{n}^{\prime}}{\lambda_{n}}}+\sqrt{\frac{\lambda_{n}}{\lambda_{n}^{\prime}}}\right) .
$$

Now, let Riem be the space of all ( $D-1)$-dimensional, compact Riemannian geometries without boundaries. We then consider a space $\left(\operatorname{Riem}, d_{N}\right) / \sim$, viz. the space Riem equipped with the measure of closeness $d_{N}$ (Eq.(3)) along with a natural identification $(\sim)$ of isospectral manifolds.]

It is proved that the space of all geometries $\left(\right.$ Riem, $\left.d_{N}\right) / \sim$ forms a metrizable space [4. It means that the measure of closeness $d_{N}$ (Eq.(3) ) can be regarded as if it were a distance in spite of its mild violation of the triangle inequality [7, 4]. From now on, thus, let us call $d_{N}\left(\mathcal{G}, \mathcal{G}^{\prime}\right)$ in Eq. (3) the spectral distance of order $N$ between two geometries $\mathcal{G}$ and $\mathcal{G}^{\prime}$. Let $\mathcal{S}_{N}$ be the completion of $\left(\right.$ Riem, $\left.d_{N}\right) / \sim$ by means of the latter's metrizable structure, since such a complete space is mathematically more desirable. One might call $\mathcal{S}_{N}$ the space of all spaces of order $N$, or the spectral space of geometries of order $N$. Mainly due to its metrizable nature, $\mathcal{S}_{N}$ possesses several nice properties such as the second countability, paracompactness (then the partition of unity can be introduced on $\mathcal{S}_{N}$ ) and locally-compactness (then an integral over $\mathcal{S}_{N}$ can be defined). ㅇ

When we focus on the discrepancy of two geometries that are very close to each other in $\mathcal{S}_{N}$, there is no significant difference even if we replace $\mathcal{F}_{1}(x)$ (used in Eq.(3) ) with some other continuous function $\mathcal{F}(x)$ satisfying the conditions in (2) (see Eq.(10) below and the arguments there on this point).

Let us introduce some convenient notations for later use. Let $\mathcal{A}$ and $\mathcal{A}_{a b}$ be any function and any symmetric tensor field, respectively, on a spatial geometry

\footnotetext{
${ }^{7}$ When two Riemannian manifolds possess the identical spectra of the Laplacian even though they are not isometric, they are said to be isospectral to each other. There are some known examples of the isospectral manifolds. For more details, see Refs. [10] and 11. For the physical interpretation of isospectral manifolds, see Refs. [6] and [7].

8 It turns out that 国 the distance function for metrization is provided by Eq.(11) with the choice $\mathcal{F}_{0}(x):=\frac{1}{2} \ln \max (\sqrt{x}, 1 / \sqrt{x})$ for $\mathcal{F}$.

${ }^{9}$ For basic facts of point set topology, see e.g. Refs. [12] and [13].
} 
$(\Sigma, h)$. Then, we define spatial-diffeomorphism invariant quantities $\langle\mathcal{A}\rangle_{n}$ and $\left\langle\mathcal{A}_{a b}\right\rangle_{n}$ as

$$
\langle\mathcal{A}\rangle_{n}:=\int_{\Sigma} f_{n} \mathcal{A} f_{n} \sqrt{ },\left\langle\mathcal{A}_{a b}\right\rangle_{n}:=\frac{1}{\lambda_{n}} \int_{\Sigma} \partial^{a} f_{n} \mathcal{A}_{a b} \partial^{b} f_{n} \sqrt{ },
$$

where $f_{n}$ is the $n$-th eigenfunction. For a quantity $\left\langle\mathcal{A}_{a b}\right\rangle_{n}$, it is assumed $n \geq 1$.

Any function $\mathcal{A}(\cdot)$ on the spatial section $\Sigma$ can be expanded in terms of $\left\{f_{n}\right\}_{n=0}^{\infty}$, such as $\mathcal{A}(\cdot)=\sum_{n=0}^{\infty} \mathcal{A}_{n} f_{n}(\cdot)$. Here we note

$$
\mathcal{A}_{n}=\left(f_{n}, \mathcal{A}\right):=\int_{\Sigma} f_{n} \mathcal{A} \sqrt{ } .
$$

The 0 -th component $\mathcal{A}_{0}$ is related to the spatial average of $\mathcal{A}$ over $\Sigma, \mathcal{A}_{\mathrm{av}}:=$ $\frac{1}{V} \int_{\Sigma} \mathcal{A} \sqrt{ }$, as

$$
\mathcal{A}_{\text {av }}=\mathcal{A}_{0} / \sqrt{V},
$$

where $V$ is the $(D-1)$-volume of the spatial section $(\Sigma, h)$.

We also introduce the quantity

$$
(l m n):=\left\langle f_{m}\right\rangle_{l n}=\int f_{l} f_{m} f_{n} \sqrt{ },
$$

which is totally symmetric in $l, m$ and $n$.

It is useful to define $\epsilon_{a b}$ and $r_{a b}$, the trace-free components of the extrinsic curvature $K_{a b}$ and the Ricci tensor $\boldsymbol{R}_{a b}$ for $(\Sigma, h)$, respectively;

$$
\epsilon_{a b}:=K_{a b}-\frac{1}{D-1} K h_{a b}, r_{a b}:=\boldsymbol{R}_{a b}-\frac{1}{D-1} \boldsymbol{R} h_{a b} .
$$

These quantities describe anisotropy of the geometry $(\Sigma, h)$.

At this stage it is appropriate to make it clear what the terms "the real Universe" ("reality") and "the model" actually indicate in this paper. We shall compare two spaces, $\mathcal{G}$ and $\mathcal{G}^{\prime}$, that are very close to each other in $\mathcal{S}_{N}$; this is a totally well-defined situation without any ambiguity. Throughout this paper, we deal with only this mathematically well-posed problem. Only on the final stage, however, we may interpret the results of this analysis in the context of the averaging problem in cosmology, supposing that one of the spaces corresponds to the real Universe and the other to its model. To keep in mind this final interpretation, we symbolically refer to the simpler space among the $\mathcal{G}$ and $\mathcal{G}^{\prime}$ as the "model", while the other space as the "real Universe" ("reality").

It is now in principle possible to investigate the evolution of the discrepancy between the reality and its model generally. In this paper, however, we restrict ourselves to the cases when

(A1) the discrepancy between $\mathcal{G}$ and $\mathcal{G}^{\prime}$ can be totally described in terms of the difference in the spatial metric, and 
(A2) they are initially so close to each other in $\mathcal{S}_{N}$ that the linear treatment of the discrepancy is justified at least during a certain period of time.

(The assumption (A1) implies that the two spaces possess the identical global topology.)

Now, let us consider two nearby spaces $\mathcal{G}$ and $\mathcal{G}^{\prime}$ in $\mathcal{S}_{N}$; their discrepancy is represented by a small difference in their spatial metrics,

$$
\gamma_{a b}:=h_{a b}^{\prime}-h_{a b},
$$

where $h_{a b}$ and $h_{a b}^{\prime}$ are the spatial metrics for $\mathcal{G}$ and $\mathcal{G}^{\prime}$, respectively. We treat $\gamma_{a b}$ as a small quantity and indicate its order of magnitude by $O(\gamma)$.

By means of the variation formulas for the spectra [5], we get

$$
\delta \ln \lambda_{n}:=\frac{\lambda_{n}^{\prime}-\lambda_{n}}{\lambda_{n}}=-\left\langle\bar{\gamma}_{a b}\right\rangle_{n}-\frac{1}{2}\langle\gamma\rangle_{n},
$$

where $\gamma:=h^{a b} \gamma_{a b}$ and $\bar{\gamma}_{a b}:=\gamma_{a b}-\frac{1}{2} \gamma h_{a b}$.

Since Eq.(8) plays the role of our key equation, let us briefly review how to derive it. The discrepancy of metrics, $\gamma_{a b}:=h_{a b}^{\prime}-h_{a b}$, causes the variation of the Laplacian, $\delta \Delta$, which induces the discrepancy of the $n$-th spectrum, $\delta \lambda_{n}:=$ $\lambda_{n}^{\prime}-\lambda_{n}$. First we recall there is a well-known relation ("Fermi's golden rule")

$$
\delta \lambda_{n}=-\langle\delta \Delta\rangle_{n}:=-\int_{\Sigma} f_{n} \delta \Delta f_{n} \sqrt{ } .
$$

Thus we need the expression for $\delta \Delta$. For this purpose we take the variation of both sides of $\Delta f=\sqrt{ }^{-1} \partial_{a}\left(h^{a b} \partial_{b} f \sqrt{ }\right)$ with respect to $h_{a b}$, yielding

$$
\delta \Delta f=\frac{1}{2} \partial_{a} \gamma \partial^{a} f-\frac{1}{\sqrt{ }} \partial_{a}\left(\gamma_{c}^{a}{ }_{c}^{c} f \sqrt{ }\right) .
$$

Thus we get,

$$
\langle\delta \Delta\rangle_{n}=\frac{1}{2} \int f_{n} \partial_{a} \gamma \partial^{a} f_{n} \sqrt{ }-\int f_{n} \partial_{a}\left(\gamma^{a}{ }_{c} \partial^{c} f_{n} \sqrt{ }\right) .
$$

By suitable partial integrals, this expression along with Eq.(9) yields Eq.(8).

Let us now consider the general form, Eq.(1). We insert $\lambda_{n}^{\prime}=\lambda_{n}+\delta \lambda_{n}$ into the R.H.S. (right-hand side) and expand it in terms of $\delta \lambda_{n}$. With the help of Eq.(8), then, the leading term in Eq.(11) becomes [5]

$$
d_{N}\left(\mathcal{G}, \mathcal{G}^{\prime}\right)=\frac{1}{2} \mathcal{F}^{\prime \prime}(1) \sum_{n=1}^{N}\left(\left\langle\bar{\gamma}_{a b}\right\rangle_{n}+\frac{1}{2}\langle\gamma\rangle_{n}\right)^{2} .
$$

We thus observe a prominent feature; whenever $\mathcal{G}$ and $\mathcal{G}^{\prime}$ are very close to each other in $\mathcal{S}_{N}$, the leading term of the spectral distance $d_{N}\left(\mathcal{G}, \mathcal{G}^{\prime}\right)$ is universally 
given by Eq.(10), irrespective either of the detailed form of the spectral distance or of the gravity theory.

We note that the choice of the function $\mathcal{F}(x)$ affects only the unimportant numerical coefficient of $d_{N}\left(\mathcal{G}, \mathcal{G}^{\prime}\right)$ in the leading order, irrespective of the cut-off order $N$. Thus, it suffices to consider a particular form given by Eq.(3). Then $\mathcal{F}^{\prime \prime}(1)=\frac{1}{8}$, so that Eq.(10) becomes

$$
d_{N}\left(\mathcal{G}, \mathcal{G}^{\prime}\right)=\frac{1}{16} \sum_{n=1}^{N}\left(\left\langle\bar{\gamma}_{a b}\right\rangle_{n}+\frac{1}{2}\langle\gamma\rangle_{n}\right)^{2}=\frac{1}{16} \vec{\gamma} \cdot \vec{\gamma}
$$

where in the last line, $\vec{\gamma}$ indicates a vector in $\mathbf{R}^{N}$ whose $n$-th component is $\gamma_{n}:=\left\langle\bar{\gamma}_{a b}\right\rangle_{n}+\frac{1}{2}\langle\gamma\rangle_{n}$. We can further derive the expressions for $\dot{d}_{N}\left(\mathcal{G}, \mathcal{G}^{\prime}\right)$ and $\ddot{d}_{N}\left(\mathcal{G}, \mathcal{G}^{\prime}\right)$ (For more details, see Ref. [5]).

Equation Eq.(11) plays the role of the central equation for our investigation on the evolution of the discrepancy between $\mathcal{G}$ and $\mathcal{G}^{\prime}$.

\section{Quantitative description of the relation be- tween two nearby geometries}

\subsection{Models to be investigated}

Let us now investigate explicitly the discrepancy of two specific nearby geometries. ${ }^{\text {.0 }}$

As a "model" universe, we construct a spacetime in the form of $T^{3} \times \mathbf{R}$ as

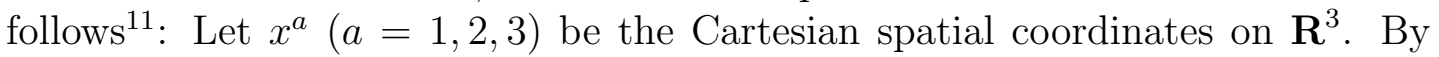
imposing the identification $x^{a} \sim x^{a}+1(a=1,2,3)$, then, $x^{a}(a=1,2,3)$ turns to a coordinate on a circle $S^{1}$, taking its value in $[0,1]$ with the identification $0 \sim 1$. We then consider a metric defined by

$$
d s^{2}=-d t^{2}+a(t)^{2} \delta_{a b} d x^{a} d x^{b},
$$

where $a(t)$ is the scale-factor of the model. The $t=$ constant spatial section of this model, $\Sigma_{t}$, corresponds to $\mathcal{G}$ in the previous section.

As for the "real Universe", a perturbed geometry around the above model is chosen: We prepare a spacetime $T^{3} \times \mathbf{R}$ with the same spatial coordinates as above, $x^{\prime a} \in[0,1]$ with $0 \sim 1$. The metric is then given by

$$
d s^{2}=-d t^{2}+h_{a b} d x^{\prime a} d x^{\prime b},
$$

\footnotetext{
${ }^{10}$ We set $D=4$ hereafter unless otherwise stated.

${ }^{11}$ In this paper, Roman indices (such as $a, b, \cdots, k, l, \cdots$ ) indicate the spatial indices and they are raised and lowered by the spatial metric. On the other hand, Greek indices (such as $\alpha$, $\beta, \cdots)$ indicate the spacetime indices and they are raised and lowered by the spacetime metric.
} 
where the spatial metric $h_{a b}$ is given by

$$
\begin{aligned}
h_{a b}\left(t, x^{\prime a}\right) & =a^{2}(t) \tilde{h}_{a b}\left(t, x^{\prime a}\right):=a^{2}(t)\left(\delta_{a b}+\tilde{\gamma}_{a b}\left(t, x^{\prime a}\right)\right), \\
\gamma_{a b}\left(t, x^{\prime a}\right): & =a^{2}(t) \tilde{\gamma}_{a b}\left(t, x^{\prime a}\right) .
\end{aligned}
$$

The $t=$ constant spatial section of this spacetime, $\Sigma_{t}^{\prime}$, corresponds to $\mathcal{G}^{\prime}$ in the previous section.

For later convenience, let $T^{3}(1)$ denote the regular 3 -torus that is conformally equivalent to $\mathcal{G} ; T^{3}(1):=[0,1] \times[0,1] \times[0,1] / \sim$, with the standard metric $d l^{2}=\delta_{a b} d x^{a} d x^{b}$ (/ $\sim$ indicates the identification for making the regular 3-torus). Let $\left\{\tilde{\lambda}_{n}\right\}_{n=0,1,2, \cdots}$ and $\left\{\tilde{f}_{n}\right\}_{n=0,1,2, \ldots}$ are, respectively, the spectra and the eigenfunctions of the Laplacian on $T^{3}(1), \tilde{\Delta}:=\delta^{a b} \partial_{a} \partial_{b}$.

\subsection{Spectral representation of geometrical quantities}

In view of Eqs.(A7), (A11) and (A16), the metric perturbation $\tilde{\gamma}_{a b}$ in Eq.(14) can be expanded in terms of $\tilde{f}_{A} \tilde{h}_{a b}, \tilde{\sigma}_{a b}^{A}, \tilde{\zeta}_{a b}^{A}$ and $\tilde{w}_{a b}^{A}$ :

$$
\tilde{\gamma}_{a b}=\frac{1}{3} \sum_{A} \gamma_{A}^{(C)} \tilde{f}_{A} \tilde{h}_{a b}+\sum_{A}^{\prime} \gamma_{A}^{(S)} \tilde{\sigma}_{a b}^{A}+\sum_{A}^{\prime} \gamma_{A}^{(V)} \tilde{\zeta}_{a b}^{A}+\sum_{A} \gamma_{A}^{(T)} \tilde{w}_{a b}^{A}
$$

Here the prime symbol in $\sum_{A}{ }^{\prime}$ implies omitting the zero-mode of $\tilde{\Delta}$ from the summation; the coefficients $\gamma_{A}^{(C)}, \gamma_{A}^{(S)}, \gamma_{A}^{(V)}$ and $\gamma_{A}^{(T)}$ are attributed to the perturbations of conformal-type, scalar-type, vector-type and tensor-type, respectively. We consider any perturbed quantity up to $O(\gamma)$ hereafter. From Eq.(15), we get

$$
\gamma=h^{a b} \gamma_{a b}=\sum_{A} \gamma_{A}^{(C)} \tilde{f}_{A}
$$

Now from Eqs.(14) and (15), it is straightforward to compute $\boldsymbol{R}_{a b}, \boldsymbol{R}$ and $r_{a b}$ (see Eq.(6i) ) for $\mathcal{G}^{\prime}$. Extracting the $f_{A^{-}}$-components of $\boldsymbol{R}$ and $D^{a} D^{b} r_{a b}$ (according to Eq.(四)), thus, we get

$$
\begin{aligned}
\boldsymbol{R}_{A} & =\frac{2}{3} \lambda_{A}\left(\gamma_{A}^{(C)}+\gamma_{A}^{(S)}\right) \sqrt{a^{3}} \quad(A \neq 0), \\
\left(D^{a} D^{b} r_{a b}\right)_{A} & =-\frac{1}{9} \lambda_{A}\left(\gamma_{A}^{(C)}+\gamma_{A}^{(S)}\right) \sqrt{a^{3}}(A \neq 0) .
\end{aligned}
$$

The two formulas in (17) are equivalent to each other due to the Bianchi identity, $D^{a}\left(\boldsymbol{R}_{a b}-\frac{1}{2} \boldsymbol{R} h_{a b}\right)=0 . \mathrm{P}^{2}$

In the same manner, $K$ and $\epsilon_{a b}$ (see Eq.(6i)) are expanded as

$$
\begin{aligned}
K & =K_{\mathrm{av}}\left(1+\sum_{A}^{\prime} \kappa_{A} \tilde{f}_{A}\right), \\
\epsilon_{a b} & =K_{\mathrm{av}}\left\{\sum_{A}^{\prime}\left(a^{2} \beta_{A}^{(S)}\right) \tilde{\sigma}_{a b}^{A}+\sum_{A}^{\prime}\left(a^{2} \beta_{A}^{(V)}\right) \tilde{\zeta}_{a b}^{A}+\sum_{A}\left(a^{2} \beta_{A}^{(T)}\right) \tilde{w}_{a b}^{A}\right\} .
\end{aligned}
$$

\footnotetext{
${ }^{12}$ For, the spectral representation of the Bianchi identity becomes $\boldsymbol{R}_{A}+\frac{6}{\lambda_{A}}\left(D^{a} D^{b} r_{a b}\right)_{A}=0$ $(A \neq 0)$ 河.
} 
Extracting the $f_{A^{-}}$-components of $K$ and $D^{a} D^{b} \epsilon_{a b}$, thus, we get

$$
\begin{aligned}
& K_{A}=\left\{\begin{array}{rr}
3 H \sqrt{V} & (A=0), \\
3 H \kappa_{A} \sqrt{a^{3}} & (A \neq 0),
\end{array}\right. \\
& \left(D^{a} D^{b} \epsilon_{a b}\right)_{A}=2 H \lambda_{A} \beta_{A}^{(S)} \sqrt{a^{3}} \quad(A \neq 0) .
\end{aligned}
$$

Here we used the relation $K_{\mathrm{av}}=3 H:=3 \dot{a} / a$; To obtain the last equation, the second equation in (A8) has been used.

\subsection{Spectral representation of matter quantities}

We consider the perfect-fluid type matter, $T^{\alpha \beta}=(\rho+p) u^{\alpha} u^{\beta}+p g^{\alpha \beta}$, where $u^{\alpha}$ is the 4 -velocity of the fluid element $\left(u^{\alpha} u_{\alpha}=-1\right)$. The matter velocity, $u^{a}$ $(a=1,2,3)$, is regarded as $O(\gamma)$. The standard parameters are introduced for later use: $\nu:=p / \rho(0 \leq \nu \leq 1 / 3$ for normal matter $)$ and $c_{s}:=\sqrt{d p / d \rho}$, the sound velocity of the matter relative to the fluid element.

We expand $\rho$ and $p$ as

$$
\rho=\rho_{\mathrm{av}}(1+\delta), \quad p=p_{\mathrm{av}}+\rho_{\mathrm{av}} c_{s}^{2} \delta, \quad \delta=\sum_{A}^{\prime} \delta_{A} \tilde{f}_{A}
$$

Then, the spectral representation of $\rho$ becomes

$$
\rho_{A}=\left\{\begin{aligned}
\rho_{\mathrm{av}} \sqrt{V} & (A=0) \\
\delta_{A} \rho_{\mathrm{av}} \sqrt{a^{3}} & (A \neq 0) .
\end{aligned}\right.
$$

The expansion of matter $\theta:=D_{a} u^{a}$ is also expanded as $\theta=3 H \sum_{A}{ }^{\prime} \Theta_{A} \tilde{f}_{A}$, then its spectral representation becomes

$$
\theta_{A}=3 H \Theta_{A} \sqrt{a^{3}} \quad(A \neq 0) .
$$

Then the divergence of the momentum density of matter, $J_{a}=\left(\rho_{a v}+p_{a v}\right) u_{a}+$ $O\left(\gamma^{2}\right)$, reduces to the spectral representation,

$$
(\vec{D} \cdot \vec{J})_{A} \simeq\left(\rho_{\mathrm{av}}+p_{\mathrm{av}}\right) \theta=3(1+\nu) H \rho_{\mathrm{av}} \Theta_{A} \sqrt{a^{3}} \quad(A \neq 0) .
$$

\subsection{Spectral representation of constraint equations}

The Hamiltonian constraint is represented in the spectral form as [5]

$$
\boldsymbol{R}_{A}+\frac{2}{3} \sum_{A^{\prime} A^{\prime \prime}} K_{A^{\prime}} K_{A^{\prime \prime}}\left(A^{\prime} A^{\prime \prime} A\right)-\frac{1}{\alpha} \rho_{A}-2 \Lambda \sqrt{V} \delta_{A 0}-\left(\epsilon_{a b} \epsilon^{a b}\right)_{A}=0
$$

where $\alpha:=\frac{c^{3}}{16 \pi G}$. (See Eq.(5) for the definition of $\left(A^{\prime} A^{\prime \prime} A\right)$.) We neglect the quantities of order $O\left(\gamma^{2}\right)$. Then, for $A=0$, Eq.(24) becomes

$$
6 H^{2}-\frac{1}{\alpha} \rho_{\mathrm{av}}-2 \Lambda=0
$$


with the help of Eqs.(19) and (21). (Here we note $\left(A^{\prime} A^{\prime \prime} 0\right)=\frac{1}{\sqrt{V}} \delta_{A^{\prime} A^{\prime \prime}}$.) Needless to say, Eq.(25) coincides with the standard equation for the (locally) flat Friedmann-Robertson-Walker model. On the other hand, Eq.(24) becomes, for $A \neq 0$,

$$
\frac{2}{3} \lambda_{A}\left(\gamma_{A}^{(C)}+\gamma_{A}^{(S)}\right)+12 H^{2} \kappa_{A}-\frac{1}{\alpha} \rho_{\mathrm{av}} \delta_{A}=0 \quad(A \neq 0),
$$

with the help of Eqs.(17), (19) and (21). (Here we note $\sum_{A^{\prime} A^{\prime \prime}} K_{A^{\prime}} K_{A^{\prime \prime}}\left(A^{\prime} A^{\prime \prime} A\right)=$ $18 H^{2} \kappa_{A} \sqrt{a^{3}}+O\left(\gamma^{2}\right)$ for $A \neq 0$.) With the help of Eq.(25), Eq.(26) is represented as

$$
\gamma_{A}^{(C)}+\gamma_{A}^{(S)}=\frac{9 H^{2}}{\lambda_{A}}\left\{\left(1-\frac{\Lambda}{3 H^{2}}\right) \delta_{A}-2 \kappa_{A}\right\} \quad(A \neq 0) .
$$

On the other hand, the momentum constraint in the spectral form becomes [5]

$$
K_{A}+\frac{3}{2} \frac{1}{\lambda_{A}}\left\{\left(D^{a} D^{b} \epsilon_{a b}\right)_{A}+\frac{1}{2 \alpha}(\vec{D} \cdot \vec{J})_{A}\right\}=0 \quad(A \neq 0) .
$$

With the help of Eqs.(19) and (23), thus, Eq.(28) gives

$$
\left(\kappa_{A}+\beta_{A}^{(S)}\right)+\frac{3}{2} \frac{1}{\lambda_{A}}(1+\nu)\left(3 H^{2}-\Lambda\right) \Theta_{A}=0 \quad(A \neq 0),
$$

where Eq.(25) was used to eliminate $\rho_{\text {av }}$.

The coefficients $\kappa_{A}$ 's and $\beta_{A}$ 's are related to $\dot{\gamma}_{A}$ 's: From Eqs. (13) and (14), we get $K_{a b}=\frac{1}{2} \dot{h}_{a b}=\frac{\dot{a}}{a} h_{a b}+\frac{a^{2}}{2} \dot{\tilde{\gamma}}_{a b}$. Together with Eqs. (15) and (16), this relation yields the spectral expressions for $K$ and $\epsilon_{a b}$ up to $O(\gamma)$. Comparing them with Eq.(18), we finally get

$$
\begin{aligned}
& \dot{\gamma}_{0}^{(C)}=6\left(H-\frac{\dot{a}}{a}\right)=: 6 \delta H, \quad \kappa_{A}=\frac{1}{6 H} \dot{\gamma}_{A}^{(C)}(A \neq 0), \\
& \beta_{A}^{(S)}=\frac{1}{6 H} \dot{\gamma}_{A}^{(S)}(A \neq 0), \quad \beta_{A}^{(V)}=\frac{1}{6 H} \dot{\gamma}_{A}^{(V)}(A \neq 0), \quad \beta_{A}^{(T)}=\frac{1}{6 H} \dot{\gamma}_{A}^{(T)} .
\end{aligned}
$$

Then Eq.(29) reduces to

$$
\left(\gamma_{A}^{(C)}+\gamma_{A}^{(S)}\right)^{\cdot}+\frac{9 H}{\lambda_{A}}(1+\nu)\left(3 H^{2}-\Lambda\right) \Theta_{A}=0 \quad(A \neq 0) .
$$

In particular, we note that $\gamma_{A}^{(C)}+\gamma_{A}^{(S)}=$ constant when $\theta=0$.

We have obtained necessary relations to investigate $\gamma_{A}:=\left\langle\bar{\gamma}_{a b}\right\rangle_{A}+\frac{1}{2}\langle\gamma\rangle_{A}$, i.e. the $A$-th component of $\vec{\gamma}$, which is important in view of Eq.(11). With the help of Eqs.(15), (16), (A5), (A8), (A12) and (A17), we get after some manipulations,

$$
\gamma_{A}=\frac{1}{3} \gamma_{0}^{(C)}+\frac{1}{3} \sum_{A^{\prime}}^{\prime}\left(c_{A A^{\prime}}^{(1)}+c_{A A^{\prime}}^{(2)}\right)\left(\gamma_{A^{\prime}}^{(C)}+\gamma_{A^{\prime}}^{(S)}\right)+\sum_{A^{\prime}} c_{A A^{\prime}}^{(T)} \gamma_{A^{\prime}}^{(T)}
$$

where $c_{A A^{\prime}}^{(1)}, c_{A A^{\prime}}^{(2)}$ and $c_{A A^{\prime}}^{(T)}$ are given in Eqs. (A5) and (A17). We note that the vector components do not appear in Eq.(32); it can be traced back to the spatialdiffeomorphism invariance of the spectral distance (Eq.(1D)). Thus we can safely omit the vector quantities (those with suffix $(V)$ ) hereafter. 


\section{Combination of the spectral scheme and the linear structure-formation theory}

Looking at Eq.(32), the behavior of coefficients $\gamma_{A}^{(C)}, \gamma_{A}^{(S)}$ and $\gamma_{A}^{(T)}$ determine the evolution of the spectral distance $d_{N}\left(\mathcal{G}, \mathcal{G}^{\prime}\right)$. Considering Eq. (15), these behaviors are translated from the time evolution of $\tilde{\gamma}_{a b}$, which can be estimated by the structure-formation theory. (The basic results of the standard linear structureformation theory is summarized in Appendix B [14].) Here two scales control the situation: The physical wave-length of the perturbation (of geometry and matter), $\lambda_{\text {phys }}$, and the causal scale at the time $t, c_{s} t\left(c_{s}\right.$ is the sound velocity of matter).

\subsection{Behavior of coefficients $\gamma_{A}, \delta_{A}, \beta_{A}$ and $\kappa_{A}$ : The case of $\lambda_{\text {phys }} \gg c_{s} t$ and $\theta \neq 0$}

We first consider the case (I) $\lambda_{\text {phys }} \gg c_{s} t$ and $\theta \neq 0$.

Since $\frac{\lambda_{\text {phys }}}{c_{s}} \gg t$ in this case, a perturbation mode satisfying this condition does not oscillate in effect during the cosmological time-scale $t$. Comparing the expansions of $\gamma, \delta$ and $\theta$ in Eqs.(16), (20) and (22) with their behavior in Eq.(B1), we see that

$$
\delta_{A}(t) \propto t^{\frac{9 \nu-1}{3(1+\nu)}}, \quad \gamma_{A}^{(C)}(t)=-\frac{2(1+3 \nu)}{\nu(9 \nu-1)} \delta_{A}, \quad \Theta_{A}(t)=\frac{(1-\nu)(6 \nu+1)}{6 \nu(1+\nu)} \delta_{A},
$$

where we have used $H(t)=\frac{2}{3(1+\nu)} 1 / t+O(\gamma)$ (the standard result derived from Eq.(25)) in the last equation.

On the other hand, comparing the expansion of $\tilde{\gamma}_{k l}$ in Eq.(15) with their behavior in Eq.(B2), we get

$$
\gamma_{A}^{(S)}(t), \gamma_{A}^{(T)}(t) \propto \frac{1}{\nu}\left(\frac{a^{2}}{k^{2} t^{2}}\right) \delta_{A}(t) \propto \frac{1}{\nu}\left(\frac{\lambda_{\text {phys }}}{c_{s} t}\right)^{2} \delta_{A}(t) \propto \frac{1}{\nu k^{2}} t^{-\frac{1-\nu}{1+\nu}} .
$$

We omit vector quantities since they do not contribute to the behavior of the spectral distance. Since $\frac{\lambda_{\text {phys }}}{c_{s} t} \gg 1$, Eqs.(33) and (34) indicate

$$
\left|\gamma_{A}^{(C)}\right| \ll\left|\gamma_{A}^{(S)}\right|,\left|\gamma_{A}^{(T)}\right|
$$

which is compatible with Eq.(B3).

Now from Eq.(30) we see that

$$
\kappa_{A}=\frac{1}{6 H} \dot{\gamma}_{A}^{(C)} \propto \gamma_{A}^{(C)} \propto \frac{1}{\nu} t^{\frac{9 \nu-1}{3(1+\nu)}}
$$

where we have used $\left|\dot{\gamma}_{A}^{(C)}\right| \propto\left|\gamma_{A}^{(C)}\right| / t$ since $t \ll \tau$ (see Appendix B.1) and $H \propto 1 / t$. 
In the same manner, from Eq.(30),

$$
\begin{aligned}
& \beta_{A}^{(S)} \propto \gamma_{A}^{(S)} \propto \frac{1}{\nu}\left(\frac{\lambda_{\text {phys }}}{c_{s} t}\right)^{2} \delta_{A} \propto \frac{1}{\nu k^{2}} t^{-\frac{1-\nu}{1+\nu}} \\
& \beta_{A}^{(T)} \propto \gamma_{A}^{(T)} \propto \frac{1}{\nu k^{2}} t^{-\frac{1-\nu}{1+\nu}} .
\end{aligned}
$$

Comparing these coefficients with $\delta_{A}$ we see that

$$
\left(\left|\delta_{A}\right| \sim\left|\gamma_{A}^{(C)}\right| \sim\left|\kappa_{A}\right|\right) \ll\left(\left|\gamma_{A}^{(S)}\right| \sim\left|\beta_{A}^{(S)}\right|,\left|\gamma_{A}^{(T)}\right| \sim\left|\beta_{A}^{(T)}\right|\right)
$$

\subsection{Behavior of coefficients $\gamma_{A}, \delta_{A}, \beta_{A}$ and $\kappa_{A}$ : The case of $\lambda_{\text {phys }} \gg c_{s} t$ and $\theta=0$}

We next consider the case (I') $\lambda_{\text {phys }} \gg c_{s} t$ and $\theta=0$.

Comparing the expansions of $\gamma$ and $\delta$ in Eqs.(16) and (20) with their behavior in Eq. $(\mathbb{B} 4)$, we see that

$$
\delta_{A}(t) \propto t^{\frac{2(1+3 \nu)}{3(1+\nu)}}, \quad \gamma_{A}^{(C)}(t)=-\frac{2}{1+\nu} \delta_{A}
$$

Next, comparing the expansion of $\tilde{\gamma}_{k l}$ in Eq.(15) with their behavior in Eq.(B5), we get

$$
\gamma_{A}^{(S)}(t), \gamma_{A}^{(T)}(t) \propto\left(\frac{a^{2}}{k^{2} t^{2}}\right) \delta_{A}(t) \propto\left(\frac{\lambda_{\text {phys }}}{c_{s} t}\right)^{2} \delta_{A}(t) \propto \frac{1}{k^{2}} t^{0} .
$$

From Eqs.(38) and (39), we observe that

$$
\left|\gamma_{A}^{(C)}\right| \ll\left|\gamma_{A}^{(S)}\right|,\left|\gamma_{A}^{(T)}\right|
$$

which is compatible with Eq.(B7)

In the same manner as in $\$ 4.1$, we get from Eq.(30) that

$$
\begin{aligned}
\kappa_{A} & =\frac{1}{6 H} \dot{\gamma}_{A}^{(C)} \propto \gamma_{A}^{(C)} \propto t^{\frac{2(1+3 \nu)}{3(1+\nu)}} \\
\beta_{A}^{(S)} & =\frac{1}{6 H} \dot{\gamma}_{A}^{(S)} \propto \nu \delta_{A} \propto \nu t^{\frac{2(1+3 \nu)}{3(1+\nu)}}, \quad \beta_{A}^{(T)}=\frac{1}{6 H} \dot{\gamma}_{A}^{(T)} \propto \nu t^{\frac{2(1+3 \nu)}{3(1+\nu)}}
\end{aligned}
$$

where we note $H^{-1} \dot{\gamma}_{A}^{(S)}, H^{-1} \dot{\gamma}_{A}^{(T)} \propto \nu \delta_{A}$ due to Eq.(B6). Comparing Eqs.(38)-(40) with each other, we thus get

$$
\begin{gathered}
\left(\left|\delta_{A}\right| \sim\left|\gamma_{A}^{(C)}\right|\right) \sim\left(\left|\kappa_{A}\right| \sim\left|t \dot{\gamma}_{A}^{(C)}\right|\right) \sim\left(\left|\beta_{A}^{(S)}\right| \sim t\left|\dot{\gamma}_{A}^{(S)}\right|\right),\left(\left|\beta_{A}^{(T)}\right| \sim t\left|\dot{\gamma}_{A}^{(T)}\right|\right) \\
\ll\left|\gamma_{A}^{(S)}\right|,\left|\gamma_{A}^{(T)}\right| .
\end{gathered}
$$




\subsection{Behavior of coefficients $\gamma_{A}, \delta_{A}, \beta_{A}$ and $\kappa_{A}$ : The case of $\lambda_{\text {phys }} \ll c_{s} t$}

Finally, we consider the case (II) $\lambda_{\text {phys }} \ll c_{s} t$. A perturbation mode satisfying this condition oscillates many times within the cosmological time-scale.

Comparing the expansions of $\gamma, \delta$ and $\theta$ in Eqs.(16), (20) and (22) with their behavior in Eq.(B8), we observe that

$$
\begin{aligned}
\delta_{A}(t) & \propto \frac{1}{\sqrt{k}} t^{-\frac{1-3 \nu}{3(1+\nu)}} \cdot O s c, \gamma_{A}^{(C)}(t) \simeq-\delta_{A}(t) \\
\Theta_{A}(t) & \propto \frac{t}{\tau} \frac{1}{\sqrt{k}} t^{-\frac{1-3 \nu}{3(1+\nu)}} \cdot O s c^{\prime} \simeq \sqrt{k} t^{\frac{2 \nu}{1+\nu}} \cdot O s c^{\prime}
\end{aligned}
$$

Here $O s c$ and $O s c^{\prime}$ indicate the oscillatory factors with the period $\tau=2 \pi a / k c_{s}$ whose relative phase-difference is $\pi / 2$; we have used $H \propto 1 / t$ and assumed $c_{s} \approx$ constant to reach the final estimation in the third relation.

We now compare Eqs.(30) and (15) with Eq.(B9). For instance, we can estimate as $\left|\beta_{A}^{(S)}\right| \propto\left|H^{-1} \dot{\gamma}_{A}^{(S)}\right| \propto \frac{t}{\tau}\left|\tau \dot{\gamma}_{A}^{(S)}\right| \sim\left(\frac{\lambda_{\text {phys }}}{c_{s} t}\right)\left|\delta_{A}\right| \ll\left|\delta_{A}\right|$. In this manner, we get

$$
\left(\left|\delta_{A}\right| \sim\left|\gamma_{A}^{(C)}\right|\right) \sim\left(\left|\gamma_{A}^{(S)}\right|,\left|\gamma_{A}^{(T)}\right|\right) \gg\left(\left|\kappa_{A}\right| \sim\left|\beta_{A}^{(S)}\right|,\left|\beta_{A}^{(T)}\right|\right)
$$

\section{Evolution of the discrepancy between two nearby geometries}

\subsection{Some useful propositions}

Let us prepare propositions that become useful soon.

We first introduce some notations. Let $\vec{V}=\left(V_{1}, V_{2}, V_{3}\right)$ and $\vec{W}=\left(W_{1}, W_{2}, W_{3}\right)$ be vectors, whose components are non-negative integers, viz. $\vec{V}, \vec{W} \in \mathbf{N}_{0}{ }^{3}$ (where $\left.\mathbf{N}_{0}:=\{0\} \cup \mathbf{N}\right)$.

Now, $\#(\vec{V})$ is defined as the number of zero elements in $\vec{V} \cdot{ }^{3}$

Next, we say that $\vec{V}$ and $\vec{W}$ are compatible with each other and write $\vec{V} \sim_{\text {comp }}$ $\vec{W}$ when $\#(\vec{V})=\#(\vec{W})$ and the positions of their zero elements are identical.

Then we define $\vec{V}_{\|(\vec{W})}$ to be the component of $\vec{V}$ that is compatible with $\vec{W}$, viz. $\vec{V}_{\|(\vec{W})}:=\left(\left(1-\delta_{W_{1}, 0}\right) V_{1},\left(1-\delta_{W_{2}, 0}\right) V_{2},\left(1-\delta_{W_{3}, 0}\right) V_{3}\right)$.

Similarly, we define $\vec{V}_{\perp(\vec{W})}$ to be the component of $\vec{V}$ that is causing the incompatibility with $\vec{W}$, viz. $\vec{V}_{\|(\vec{W})}:=\vec{V}-\vec{V}_{\|(\vec{W})}=\left(\delta_{W_{1}, 0} V_{1}, \delta_{W_{2}, 0} V_{2}, \delta_{W_{3}, 0} V_{3}\right)$.

\footnotetext{
${ }^{13}$ For example, $\#(1,2,3)=0, \#(3,0,2)=1$ and $\#(0,0,0)=3$.

${ }^{14}$ For example, $(1,3,0) \sim_{\text {comp }}(2,1,0),(2,0,3) \sim_{\text {comp }}(3,0,3) ;(2,3,0) \chi_{\text {comp }}(0,1,2)$.

${ }^{15}$ For example, when $\vec{V}=(2,3,1)$ and $\vec{W}=(0,1,2), \vec{V}_{\|(\vec{W})}=(0,3,1)$.
} 
We may simply write $\vec{V}_{\|}$and $\vec{V}_{\perp}$ instead of $\vec{V}_{\|(\vec{W})}$ and $\vec{V}_{\perp(\vec{W})}$, respectively, whenever obvious.

As is explained in Appendix A, the basic modes of $\Delta$ on the model space $\Sigma$ are labeled by the pair of two vectors, $(\vec{n}, \vec{\sigma}),\left(n_{1}, n_{2}, n_{3} \in \mathbf{N}_{0} ; \sigma_{1}, \sigma_{2}, \sigma_{3} \in\{0,1\}\right)$. We often write simply $A$ for $(\vec{n}, \vec{\sigma})$. Note that $A=(\overrightarrow{0}, \vec{\sigma})$ automatically implies $A=(\overrightarrow{0}, \overrightarrow{0})$ since $f_{\vec{n}=\overrightarrow{0}}^{(\sigma=\overrightarrow{0})}=\frac{1}{\sqrt{a^{3}}}$ is the only possible eigenfunction for $\vec{n}=\overrightarrow{0}$ (see Eq.(A1) $)$.

For $A=(\vec{n}, \vec{\sigma})$ and $A^{\prime}=\left(\vec{n}^{\prime}, \vec{\sigma}^{\prime}\right)$, the quantity $\#\left(\vec{\sigma}_{\|\left(\vec{n}^{\prime}\right)}\right)$ (the number of zero components in $\vec{\sigma}_{\|\left(\vec{n}^{\prime}\right)}$ ) has just been defined above.

Now we can state the proposition. Let $c_{A A^{\prime}}$ be any function of $A=(\vec{n}, \vec{\sigma})$ and $A^{\prime}=\left(\vec{n}^{\prime}, \vec{\sigma}^{\prime}\right)$ containing the factor $(-)^{\#\left(\vec{\sigma}_{\|\left(\vec{n}^{\prime}\right)}\right)}$. Then, for $\alpha_{A}$ which is any function of $A$, it follows that

Proposition 1

$$
\sum_{A}^{\prime} c_{A A^{\prime}} \alpha_{A^{\prime}}=\left\{\begin{array}{rr}
0 & \left(\vec{n}^{\prime} \neq \overrightarrow{0}\right) \\
\alpha_{0} \sum_{A}{ }^{\prime} c_{A 0} & \left(\vec{n}^{\prime}=\overrightarrow{0}\right)
\end{array} .\right.
$$

(Here $\alpha_{0}$ and $c_{A 0}$ are abbreviations for $\alpha_{(\overrightarrow{0}, \overrightarrow{0})}$ and $c_{A(\overrightarrow{0}, \overrightarrow{0})}$, respectively; the prime symbol in $\sum_{A}{ }^{\prime}$ indicates $A=(\overrightarrow{0}, \overrightarrow{0})$ is not included in the summation.) For the proof, we note that the L.H.S. (left-hand side) contains a factor $\sum_{\vec{\sigma}}(-)^{\#\left(\vec{\sigma}_{\|\left(\vec{n}^{\prime}\right)}\right)}$. This factor is nothing but the summation of +1 's and the same number of -1 's (so it vanishes), unless “ $\vec{\sigma}_{\|\left(\vec{n}^{\prime}\right)} \equiv \overrightarrow{0}$ independently of $\vec{\sigma} " \boxplus$; it means that the L.H.S. vanishes unless $\vec{n}^{\prime}=\overrightarrow{0}$, so that the proposition follows.

As a corollary, it immediately follows that

\section{Corollary of Proposition 1}

$$
\sum_{A}^{\prime} \sum_{A^{\prime}}^{\prime} c_{A A^{\prime}} \alpha_{A^{\prime}}=0
$$

Now, we show another proposition. Let $A=(\vec{n}, \vec{\sigma})$ and $A^{\prime}=\left(\vec{n}^{\prime}, \vec{\sigma}^{\prime}\right)$ once more. It

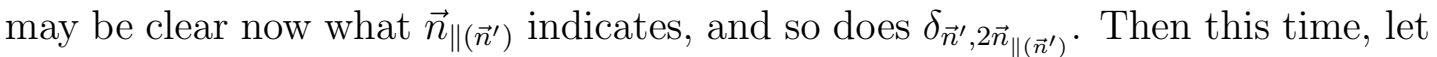
$c_{A A^{\prime}}$ and $c^{\prime}{ }_{A A^{\prime}}$ be any functions which contain the factor $(-)^{\#\left(\vec{\sigma}_{\|\left(\vec{n}^{\prime}\right)}\right)} \delta_{\vec{n}^{\prime}, 2 \vec{n}_{\|\left(\vec{n}^{\prime}\right)}} \delta_{\vec{\sigma}^{\prime}, \overrightarrow{0}^{\prime}}$. Then, for $\alpha_{A^{\prime}}$ and $\beta_{A^{\prime}}$ that are any functions of $A^{\prime}$, it follows

Proposition 2

$$
\sum_{A}^{\prime}\left(\sum_{A^{\prime}}^{\prime} c_{A A^{\prime}} \alpha_{A^{\prime}}\right)\left(\sum_{A^{\prime \prime}}^{\prime} c_{A A^{\prime \prime}}^{\prime} \beta_{A^{\prime \prime}}\right)=\sum_{A}^{\prime} \sum_{A^{\prime}}^{\prime} c_{A A^{\prime}} c_{A A^{\prime}}^{\prime} \alpha_{A^{\prime}} \beta_{A^{\prime}}
$$

\footnotetext{
${ }^{16}$ For illustration, suppose $\vec{n}^{\prime}=(1,0,0)$. Then, $\vec{\sigma}_{\|\left(\vec{n}^{\prime}\right)}=\left(\sigma_{1}, 0,0\right)$ so that $\#\left(\vec{\sigma}_{\|\left(\vec{n}^{\prime}\right)}\right)=$ 3,2 for $\sigma_{1}=0,1$, respectively; then $(-)^{\#\left(\vec{\sigma}_{\|\left(\vec{n}^{\prime}\right)}\right)}=-1,1$ for $\sigma_{1}=0,1$, respectively. Thus $\sum_{\vec{\sigma}}(-)^{\#\left(\vec{\sigma}_{\|\left(\vec{n}^{\prime}\right)}\right)}=0$.
} 
We note that the L.H.S. contains a factor $u:=\sum_{\sigma}(-)^{\#\left(\vec{\sigma}_{\|}\left(\vec{n}^{\prime}\right)\right)}(-)^{\#\left(\vec{\sigma}_{\|}\left(\vec{n}^{\prime \prime}\right)\right)}$. Every term in $u$ is 1 when $\vec{n}^{\prime} \sim_{\text {comp }} \vec{n}^{\prime \prime}$ so that $u$ becomes some (non-zero) natural number when $\vec{n}^{\prime} \sim_{\text {comp }} \vec{n}^{\prime \prime}$; on the other hand, when $\vec{n}^{\prime} \chi_{\text {comp }} \vec{n}^{\prime \prime}, u$ is the summation of +1 's and the same number of -1 's so that it vanishes. It means that the factor $u$ can be regarded as $\propto \delta_{\vec{n}^{\prime}, \vec{n}^{\prime \prime}}$ because of the presence of $\delta_{\vec{n}^{\prime}, 2 \vec{n}_{\|\left(\vec{n}^{\prime}\right)}} \delta_{\vec{n}^{\prime \prime}, 2 \vec{n}_{\|\left(\vec{n}^{\prime \prime}\right)}}$ on the L.H.S. Thus, noting the presence of another factor $\delta_{\vec{\sigma}^{\prime}, \overrightarrow{0}} \delta_{\vec{\sigma}^{\prime \prime}, \overrightarrow{0}}$ on the L.H.S., the claim follows.

Finally, suppose $c_{A A^{\prime}}$ satisfies the same condition as in Proposition 2. Then

Proposition 3

$$
\sum_{A}^{\prime}\left(\alpha_{0}+\sum_{A^{\prime}}^{\prime} c_{A A^{\prime}} \alpha_{A^{\prime}}\right)^{2}=\alpha_{0}^{2} \sum_{A}^{\prime} 1+\sum_{A}^{\prime} \sum_{A^{\prime}}^{\prime} c_{A A^{\prime}}^{2} \alpha_{A^{\prime}}^{2}
$$

It immediately follows from Proposition 1, its Corollary and Proposition 2.

\subsection{Estimating the time evolution of $d_{N}\left(\mathcal{G}, \mathcal{G}^{\prime}\right)$}

Now, let us look at Eqs.(11) and (32). With the help of Proposition 2 and Proposition 3, we can simplify the expression for $\gamma_{A}{ }^{2}$. We assume that the coefficients $\gamma_{A}^{(C)}, \gamma_{A}^{(S)}$, and $\gamma_{A}^{(T)}$ are invariant under the permutation $\tau$ of $A, \tau$ : $A \mapsto \tau(A)$. (More explicitly, $\tau:(\vec{n}, \vec{\sigma}) \mapsto(\tau(\vec{n}), \tau(\vec{\sigma}))$.) Then, we note that any term in $\vec{\gamma} \cdot \vec{\gamma}$ of the form

$$
\sum_{A}^{\prime} \sum_{A^{\prime}}^{\prime} c_{A A^{\prime}} c_{A A^{\prime}}^{(T)} \gamma_{A^{\prime}} \gamma_{A^{\prime}}^{(T)}
$$

vanishes unless $c_{A A^{\prime}}=c_{A A^{\prime}}^{(T)}$ because $c_{A A^{\prime}}^{(T)}$ is odd for the permutation $\sigma: A A^{\prime} \mapsto$ $\sigma(A) \sigma\left(A^{\prime}\right)$ (see Eq. (A18)), while other coefficients $c^{(1)}, c^{(2)}$ and $c^{(3)}$ as well as $\gamma_{A}^{(C)}$, $\gamma_{A}^{(S)}$, and $\gamma_{A}^{(T)}$ are even under the same permutation (see Eq. (A5)). In a similar argument of permutations, the contribution of $c_{A 0}^{(T)}$ to $\gamma_{A}^{2}$ is only in the form of $\sum_{A}^{\prime} c_{A 0}^{(T)^{2}} \gamma_{0}^{(T) 2}$.

Thus, we get

$$
\begin{aligned}
d_{N}\left(\mathcal{G}, \mathcal{G}^{\prime}\right) & =\frac{1}{16} \vec{\gamma} \cdot \vec{\gamma} \\
& =\frac{1}{144} N \gamma_{0}^{(C)}{ }^{2}+\frac{1}{144} \sum_{A}^{\prime} \sum_{A^{\prime}}^{\prime}\left(c_{A A^{\prime}}^{(1)}+c_{A A^{\prime}}^{(2)}\right)^{2}\left(\gamma_{A^{\prime}}^{(C)}+\gamma_{A^{\prime}}^{(S)}\right)^{2} \\
& +\frac{1}{16} \sum_{A}^{\prime} \sum_{A^{\prime}} c_{A A^{\prime}}^{(T)^{2} \gamma_{A^{\prime}}^{(T)}}{ }^{2}
\end{aligned}
$$

Here we note that $N$ is the number of eigenvalues less than $\lambda_{N}$; the contribution of the term $\sum_{A}^{\prime} c_{A 0}^{(T)^{2}} \gamma_{0}^{(T)}{ }^{2}$ is included in the last term on the R.H.S.

We pay attention to the three typical types of perturbations: 
(I) The case of $\lambda_{\text {phys }} \gg c_{s} t$ and $\theta \neq 0$

(I') The case of $\lambda_{\text {phys }} \gg c_{s} t$ and $\theta=0$

(II) The case of $\lambda_{\text {phys }} \ll c_{s} t$

For the case (I), the behavior of $d_{N}\left(\mathcal{G}, \mathcal{G}^{\prime}\right)$ is controlled by the behavior of $\left(\gamma_{A^{\prime}}^{(C)}+\right.$ $\left.\gamma_{A^{\prime}}^{(S)}\right)^{2} \simeq \gamma_{A^{\prime}}^{(S)}{ }^{2}$ and $\gamma_{A^{\prime}}^{(T)^{2}}$, due to Eq.(37). The situation is same for the case (I'), too, due to Eq.(41). On the other hand, for the case (II), the behavior of $d_{N}\left(\mathcal{G}, \mathcal{G}^{\prime}\right)$ is determined by the behavior of all $\left.\gamma_{0}^{(C)}\right)^{2},\left(\gamma_{A^{\prime}}^{(C)}+\gamma_{A^{\prime}}^{(S)}\right)^{2}$ and $\gamma_{A^{\prime}}^{(T)}$ because of Eq.(43).

These observations enable us to estimate the time-dependence of $\gamma_{A}{ }^{2}$. First, looking at the explicit expression for $c_{A A^{\prime}}$ 's (Eqs. (A5) and (A18)), the summations over $\sigma$ and $\sigma^{\prime}$ on the R.H.S. of Eq. (44) yield no essential contributions to $d_{\left(\mathcal{G}, \mathcal{G}^{\prime}\right)}$ within the present order of accuracy. Thus, we can safely replace $A$ with the coordinate wave-length $\vec{k}$ defined as $\vec{k}=2 \pi \vec{n}$. Then we may write $\gamma_{k}$ instead of $\gamma_{A}$, so that $d_{N}\left(\mathcal{G}, \mathcal{G}^{\prime}\right)$ is estimated as $d_{N}\left(\mathcal{G}, \mathcal{G}^{\prime}\right) \sim \int \gamma_{k}^{2} d \mu(k)$, where $\mu(k)$ is a suitable measure for $k$. Then the estimation of $\gamma_{k}{ }^{2}$ for the cases (I), (I') and (II) can be summarized as (see Eqs.(34), (39) and (42))

$$
\gamma_{k}^{2} \simeq\left\{\begin{aligned}
\frac{D(k)}{\nu^{2} k^{4}} t^{-\frac{2(1-\nu)}{1+\nu}} & \text { for (I) }, \\
\frac{D(k)}{k^{4}} t^{0} & \text { for (I') } \\
\frac{D(k)}{k} t^{-\frac{2(1-3(1))}{3(1+\nu)}} \cdot O s c^{2} & \text { for (II) },
\end{aligned}\right.
$$

where $D(k)$ denotes the extra $k$-dependence coming from $(a)$ the coefficients $\left(c_{A A^{\prime}}^{(1)}+c_{A A^{\prime}}^{(2)}\right)^{2}$ and $c_{A A^{\prime}}^{(T)}{ }^{2}$, and $(b)$ the spectral property of the perturbation $\tilde{\gamma}_{a b}$, i.e. the explicit value of $\gamma_{A}$ 's in Eq.(15)).

The $k$-dependence coming from $(a)$ is determined without ambiguity by the definition of $c_{A A^{\prime}}$ 's (Eqs. (A5) and (A18)). As is explained after Eqs. (A5) and (A18), the coefficients $c_{A A^{\prime}}$ 's do not depend on the magnitude $k$, but only depend

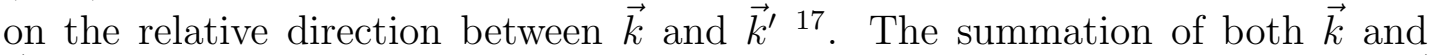
$\vec{k}^{\prime}\left(A\right.$ and $\left.A^{\prime}\right)$ in Eq.(44) ensures the summation for all the relative directions $\vec{k}$ and $\vec{k}^{\prime}$, which yields only minor corrections. The $k$-dependence coming from (b) depends on the initial condition for $\tilde{\gamma}_{a b}$. As one natural assumption for the initial condition for $\tilde{\gamma}_{a b}$, we here postulate that the $k$-dependence coming from (b) shows no peak at a particular scale but a mild $k$-dependence.

In any case $D(k)$ is not essential for the $t$-dependence of $d_{N}\left(\mathcal{G}, \mathcal{G}^{\prime}\right)$ (see arguments after Eqs.(48) and (49)). We here take care of $D(k)$ by postulating a power-law, $D(k) \propto k^{-p}$ for (I) and (I'), and $\propto k^{-q}$ for (II).

We can estimate $d_{N}\left(\mathcal{G}, \mathcal{G}^{\prime}\right)$ by converting the summation $\sum_{A}{ }^{\prime}$ in Eq. (44) to $k$-integral from $k=1$ (because of the $T^{3}$ structure of the space) up to $k=k_{N}$, the

\footnotetext{
${ }^{17}$ Note that $\vec{k}=2 \pi \vec{n}$ and $k^{2}=4 \pi^{2} \vec{n} \cdot \vec{n}$ (Eq.(A1) and below).
} 
maximum value for $k$ determined by the cut-off order $N$. (It is a standard way of estimating a summation in statistical physics, and considering Eq. (A2), this is a reasonable estimation when the universes are sufficiently large.) We consider two situations, when we are studying

(A) relatively shorter scale geometry as well as the global features of the Universe, and

(B) only the most global features of the Universe,

where the horizon scale at the time is the natural standard for "short" or "long".

Situation $(A)$ : This situation corresponds to $k_{N}>k_{H}$. Here $k_{H}:=2 \pi a / c_{s} t \propto$ $t^{-\frac{1+3 \nu}{3(1+\nu)}}$, the wave-number corresponding to the horizon scale at $t$. From $k=1$ to $k=k_{H}$ in the integral region, we can use the result for the case (I) (or (I')); from $k=k_{H}$ to $k=k_{N}$, we can use the result for the case (II). Thus we estimate, based on the case (I) for $k \in\left[1, k_{H}\right]$,

$$
\begin{aligned}
d_{N}\left(\mathcal{G}, \mathcal{G}^{\prime}\right) & \simeq A^{\prime} t^{-\frac{2(1-\nu)}{1+\nu}} \int_{1}^{k_{H}} \frac{1}{k^{4+p}} k^{2} d k+B^{\prime} t^{-\frac{2(1-3 \nu)}{3(1+\nu)}} \int_{k_{H}}^{k_{N}} \frac{1}{k^{1+q}} \cdot O s c^{2} k^{2} d k \\
& \simeq A \xi(t) t^{-\frac{2(1-\nu)}{1+\nu}}+B k_{N}^{2-q} \eta(t) t^{-\frac{2(1-3 \nu)}{3(1+\nu)}} .
\end{aligned}
$$

Here, $A$ and $B$ are appropriate positive numerical factors that make the expression dimension-free; $\xi(t):=1-C t^{\frac{(1+p)(1+3 \nu)}{1+\nu}}$ and $\eta(t):=1-C^{\prime} t^{-\frac{(2-q)(1+3 \nu)}{3(1+\nu)}}$ with $C$ and $C^{\prime}$ being appropriate positive numerical factors that make $\xi(t)$ and $\eta(t)$ are positive for $t>\exists t_{0}$. We note that $\xi(t) \rightarrow 0$ and $\eta(t) \rightarrow 1$ as $t \rightarrow \infty$. Thus as time goes on, the second term with the behavior $\sim t^{-\frac{2(1-3 \nu)}{3(1+\nu)}}$ becomes dominant.

In the same manner, based on the case (I') for $k \in\left[1, k_{H}\right]$, we estimate

$$
d_{N}\left(\mathcal{G}, \mathcal{G}^{\prime}\right) \simeq A \xi(t)+B k_{N}^{2-q} \eta(t) t^{-\frac{2(1-3 \nu)}{3(1+\nu)}}
$$

Situation (B): This situation corresponds to $k_{N}$ is less than $k_{H}$. Thus, for the case (I),

$$
\begin{aligned}
d_{N}\left(\mathcal{G}, \mathcal{G}^{\prime}\right) & \simeq A^{\prime} t^{-\frac{2(1-\nu)}{1+\nu}} \int_{1}^{k_{N}} \frac{1}{k^{4+p}} k^{2} d k \\
& \simeq A\left(1-k_{N}^{-(1+p)}\right) t^{-\frac{2(1-\nu)}{1+\nu}}
\end{aligned}
$$

In the same manner, for the case (I'), we get

$$
d_{N}\left(\mathcal{G}, \mathcal{G}^{\prime}\right) \simeq A^{\prime}\left(1-k_{N}^{-(1+p)}\right) .
$$


Looking at these results, Eqs.(46)-(49), we see that $d_{N}\left(\mathcal{G}, \mathcal{G}^{\prime}\right)$ decreases with time (with Eq.(49) being a marginal case of constancy). Typical behavior is $d_{N}\left(\mathcal{G}, \mathcal{G}^{\prime}\right) \sim t^{-\frac{2(1-3 \nu)}{3(1+\nu)}}$ for the situation $(A)$, and $\sim t^{-\frac{2(1-\nu)}{1+\nu}}$ for the situation $(B)$. It means that, for the situation $(B), d_{N}\left(\mathcal{G}, \mathcal{G}^{\prime}\right)$ decreases more rapidly than the situation (A). This might be interpreted as that, within the linear regime, the dynamics of the most global structures of the Universe are approximated by those of a model quite well, compared to the shorter-scale features.

Significantly, Eqs.(46)-(49) are also showing the scale-dependence of $d_{N}\left(\mathcal{G}, \mathcal{G}^{\prime}\right)$ as an increasing function of the cut-off scale $k_{N}$. It indicates a reasonable result that the finer structures are observed, the more discrepancy between the reality and its model is detected. This result is consistent with our observation in the previous paragraph.

Finally, we realize that $D(k)$ has no significant influence on the $t$-dependence of $d_{N}\left(\mathcal{G}, \mathcal{G}^{\prime}\right)$; rather $D(k)$ for the integral-region (II) determines the details on how $d_{N}\left(\mathcal{G}, \mathcal{G}^{\prime}\right)$ depends on the cut-off scale $N$.

We observed that, in the regime where the linear approximation of geometry is valid, $d_{N}\left(\mathcal{G}, \mathcal{G}^{\prime}\right)$ does not increase with time, rather it shows the tendency to decrease. This result is favorable to the reliability of describing the Universe approximately by means of a model, at least during some period of time. Needless to say, the analysis here is limited to just one pair of geometries within the linearregime. The present result calls for more investigations along the same line on the behavior beyond the liner-regime and wider class of spacetimes.

\section{Summary and Discussion}

In this paper, we have studied a fundamental issue rooted in the foundation of cosmology: To what extent we can rely on a model to recognize the Universe. To analyze this issue, we argued that the concept of "closeness" or "discrepancy" between two geometries was indispensable, so that we have resorted to the spectral scheme developed in the previous studies. Then we have estimated the discrepancy measured by the spectral distance between two spatial geometries, $\mathcal{G}$ and $\mathcal{G}^{\prime}$, that are very close to each other, mimicking the relation of the Universe and its model.

We have set $\mathcal{G}$ to be a flat $T^{3}$ geometry constructed from the flat FriedmannRobertson-Walker model obtained by the point-identification; as for $\mathcal{G}^{\prime}$, we have chosen the geometry generated by perturbing the geometry $\mathcal{G}$. These $\mathcal{G}$ and $\mathcal{G}^{\prime}$ were regarded as the imitation of the relation of the reality and its model.

Then the behavior of the the spectral distance $d_{N}\left(\mathcal{G}, \mathcal{G}^{\prime}\right)$ has been investigated explicitly. With the help of the linear structure-formation theory, the behavior of $d_{N}\left(\mathcal{G}, \mathcal{G}^{\prime}\right)$ has been estimated as Eqs.(46)-(49). In particular, it is an important observation that the geometry difference contributes to the discrepancy of the two universes through a particular combination $\gamma_{n}:=\left\langle\bar{\gamma}_{a b}\right\rangle_{n}+\frac{1}{2}\langle\gamma\rangle_{n}$ (Eq.(11)). 
The spectral scheme served as a powerful tool for dealing with the present problem. It is effective to analyze the type of problems involving two or more geometries. Indeed we were able to derive several non-trivial relations describing the discrepancy between the reality and its model which would have been difficult to find out without the spectral scheme. Here we realize primary importance of preparing a suitable language for talking about an entangled situation before tackling it.

We have observed that, as far as the linear approximation of geometry is valid, $d_{N}\left(\mathcal{G}, \mathcal{G}^{\prime}\right)$ does not increase with time prominently, rather it shows the tendency to decrease. This result is, at least, compatible with our belief in the validity of the approximate description of the Universe in terms of a model. The present investigation is on just one pair of geometries within the linear-regime; it is now required to investigate wider class of spacetimes as well as to study the behavior beyond the liner-regime along this line.

We have learned in this paper that it is practically possible to proceed in this direction; now we can improve the order of accuracy of approximations, e.g. the passage from Eq.(45) to Eqs.(46)-(49)). One drawback of the analysis linked with the linear structure-formation theory is that detailed features of the problem become, as it were, hidden behind it. Therefore, it is desirable to analyze the same problem via another, more basic route, i.e. by directly using the fundamental equations for $d_{N}, \dot{d}_{N}, \ddot{d}_{N}$ etc. [5].

As the next step of investigation, the evolution of $d_{N}\left(\mathcal{G}, \mathcal{G}^{\prime}\right)$ should be studied beyond the linear regime of the geometrical discrepancy. It can be done since we have fundamental relations Eqs.(3) along with the spectral evolution equations [5].

Finally we note that our approach to the averaging problem in cosmology is quite different from the "usual" one which the term "averaging" might suggest. In the spectral scheme, there is no ambiguous procedure of "averaging geometry" such as $\left\langle h_{a b}\right\rangle$ (recall the difficulty (D1) in $\S[$ (1). Rather, we first define the concept of "closeness" between geometries and choose a model $\mathcal{G}$ which is a simple geometry and reasonably close to the reality $\mathcal{G}^{\prime}$ in the space of all spaces $\mathcal{S}_{N}$. This mapping from $\mathcal{G}^{\prime}$ to $\mathcal{G}$ is what we regard "averaging" in effect. Needless to say, this procedure is mathematically well-defined. Since the concept of "closeness" $\left(d_{N}\right)$ is scale-dependent and apparatus-dependent, this mapping procedure ("averaging") is also depends on both the observational scale we are interested in and the apparatus we rely on. These are desirable properties and we can expect that the framework outlined here is glimpsing a new way of viewing spaces as scale-dependent and apparatus-dependent objects.

The type of analysis developed here has a general applicability to a wider range of theories, not restricted to cosmology. It is an issue of the internal relation of the triad (Reality, Model, Dynamics), which arises quite universally. When a theory deals with objects characterized by a sequence of real numbers ordered in an increasing order ("spectra" of the theory), $\left\{\Lambda_{n}\right\}_{n=0,1,2, \ldots}$, one can 
construct the spectral measure of closeness similar to Eq.(11) along with Eq.(2). Then one can construct the spectral space $\mathcal{S}$, which is similar to our $\mathcal{S}_{N}$. This space forms a metrizable space when the spectral measure of closeness (Eq.(目)) is chosen suitably, e.g. like Eq.(3). 18 A "good" theory should have such a property that two generic points close to each other in $\mathcal{S}$ remain close during some period of time of concern. Then we are entitled to approximate the reality by a model or classify the objects into types. The analysis presented here is the very first step for understanding the issue of types in physics. It is interesting to analyze fundamental theories from this viewpoint.

The author thanks the Ministry of Education and Science, the Government of Japan and Inamori Foundation, Japan for financial support.

\section{APPENDIX}

\section{A The basic modes}

Here we present the basic modes on the $t=$ constant spatial section, $\Sigma_{t}$, of the "model" universe $T^{3} \times \mathbf{R}$ with Eq.(12). We recall that we adopt the Cartesiantype spatial coordinates $x^{a}(a=1,2,3)$ which take the value in $[0,1]$ with the identification $0 \sim 1$. We note $\Delta=\frac{1}{a^{2}} \partial_{a} \partial_{b} \delta^{a b}$.

Now, let

$$
\cos _{\sigma} \theta:=\left\{\begin{array}{cc}
\cos \theta & (\sigma=0) \\
\sin \theta & (\sigma=1)
\end{array} .\right.
$$

Then the eigenfunctions of $\Delta$ on $\Sigma_{t}$ can be represented as

$$
\begin{aligned}
f_{\vec{n}}^{(\vec{\sigma})}\left(x_{1}, x_{2}, x_{3}\right)= & \frac{\sqrt{2^{(3-\#(\vec{\sigma}))}}}{\sqrt{a^{3}}} \cos _{\sigma_{1}} 2 \pi n_{1} x_{1} \quad \cos _{\sigma_{2}} 2 \pi n_{2} x_{2} \cos _{\sigma_{3}} 2 \pi n_{3} x_{3} \\
& \left(n_{1}, n_{2}, n_{3}=0,1,2, \cdots ; \sigma_{1}, \sigma_{2}, \sigma_{3}=0,1\right)
\end{aligned}
$$

where $\vec{\sigma}:=\left(\sigma_{1}, \sigma_{2}, \sigma_{3}\right), \vec{n}:=\left(n_{1}, n_{2}, n_{3}\right)$ and $\#(\vec{\sigma})$ denotes the number of zero elements in $\vec{\sigma}$ The corresponding spectra can be represented as

$$
\lambda_{\vec{n}}=\frac{(2 \pi)^{2}}{a^{2}} \vec{n} \cdot \vec{n}=\frac{(2 \pi)^{2}}{a^{2}}\left(n_{1}^{2}+n_{2}^{2}+n_{3}^{2}\right)
$$

\footnotetext{
${ }^{18}$ Stating more precisely, in order for the space $\mathcal{S}$ to possess desirable properties, varieties of objects in the theory should be rich enough; viz. for a given object $O$ there should always be another object $O^{\prime}$ which is an infinitesimal modification of $O$, so that the spectra for $O$ and those for $O^{\prime}$ are infinitesimally close to each other. This condition is satisfied in the case of $\mathcal{S}_{N}$; for any given geometry, there always exists its infinitesimally modified geometry [4].

${ }^{19}$ See the beginning of $\S$ f for more details on the notations such as $\#(\vec{\sigma})$.
} 
We recall that we have introduced the eigenfunction $\tilde{f}_{\vec{n}}^{(\vec{\sigma})}$ and the eigenvalue $\tilde{\lambda}_{\vec{n}}$ of the Laplacian $\tilde{\Delta}$ on the regular 3 -torus $T^{3}(1)$ (see after Eq.(12)). They are related to $f_{\vec{n}}^{(\vec{\sigma})}$ and $\lambda_{\vec{n}}$ as

$$
\tilde{f}_{\vec{n}}^{(\vec{\sigma})}:=\sqrt{a^{3}} f_{\vec{n}}^{(\vec{\sigma})}, \quad \tilde{\lambda}_{\vec{n}}:=a^{2} \lambda_{\vec{n}} .
$$

For notational simplicity, we often write $f_{A}$ and $\tilde{f}_{A}$ instead of $f_{\vec{n}}^{(\vec{\sigma})}$ and $\tilde{f}_{\vec{n}}^{(\vec{\sigma})}$, respectively, wherever explicit indication of the label $(\vec{n}, \vec{\sigma})$ can be omitted.

We recall the conformal equivalence between $\Sigma_{t}$ and $T^{3}(1)$ (see the argument after Eq.(12)). Because of this equivalence, we can define the integral over $T^{3}(1)$ for any function $\mathcal{A}$ and any symmetric tensor field $\mathcal{A}_{a b}$ on $\Sigma_{t}$,

$$
\langle\mathcal{A}\rangle_{A}:=\int_{T^{3}(1)} \tilde{f}_{A} \mathcal{A} \tilde{f}_{A} d^{3} x \quad, \quad\left\langle\mathcal{A}_{a b} \tilde{\rangle}_{A}:=\frac{1}{\tilde{\lambda}_{A}} \int_{T^{3}(1)} \partial^{a} \tilde{f}_{A} \mathcal{A}_{a b} \partial^{b} \tilde{f}_{A} d^{3} x\right.
$$

Then, it is useful to introduce the coefficients $c_{A A^{\prime}}^{(1)}, c_{A A^{\prime}}^{(2)}$ and $c_{A A^{\prime}}^{(3)}$ as

$$
\begin{aligned}
& c_{A A^{\prime}}^{(1)}:=\left\langle\tilde{f}_{A^{\prime}}\right\rangle_{A}=\sqrt{a^{3}}\left\langle f_{A^{\prime}}\right\rangle_{A} \\
& =(-)^{\#\left(\vec{\sigma}_{\|}\right)} \frac{\sqrt{2}}{4}(\sqrt{2})^{\#\left(\vec{n}^{\prime}\right)} \delta_{\vec{\sigma}^{\prime}, \overrightarrow{0}} \delta_{\vec{n}^{\prime}, 2 \vec{n}_{\|}} \delta_{\vec{n}_{\perp}^{\prime}, \overrightarrow{0}}, \\
& c_{A A^{\prime}}^{(2)}:=\left\{\begin{array}{c}
\left\langle\frac{1}{\tilde{\lambda}_{A^{\prime}}} \partial_{a} \partial_{b} \tilde{f}_{A^{\prime}}\right\rangle_{A}=\sqrt{a^{3}}\left\langle\frac{1}{\lambda_{A^{\prime}}} \partial_{a} \partial_{b} f_{A^{\prime}}\right\rangle_{A} \quad\left(\vec{n}^{\prime} \neq \overrightarrow{0}\right), \\
0 \quad\left(\vec{n}^{\prime}=\overrightarrow{0}\right)
\end{array}\right. \\
& =\hat{n}_{\|}^{2} c_{A A^{\prime}}^{(1)} \\
& c_{A A^{\prime}}^{(3)}:=-\left\langle\tilde{f}_{A^{\prime}} \tilde{h}_{a b}\right\rangle_{A}=-\sqrt{a^{3}}\left\langle f_{A^{\prime}} h_{a b}\right\rangle_{A} \\
& =\left(1-2 \hat{n}_{\perp}^{2}\right) c_{A A^{\prime}}^{(1)} \text {. }
\end{aligned}
$$

Here $\vec{n}_{\|}\left(\vec{n}_{\perp}\right)$ is, roughly speaking, the component of $\vec{n}$ parallel (perpendicular) to $\vec{n}^{\prime} \mathrm{U} ; \hat{n}_{\|}^{2}:=\left(\vec{n}_{\|} /|\vec{n}|\right)^{2}$ and $\hat{n}_{\perp}^{2}:=\left(\vec{n}_{\perp} /|\vec{n}|\right)^{2}$, so that $\hat{n}_{\|}^{2}$ and $\hat{n}_{\perp}^{2}$ possess only the information on the relative direction between $\vec{n}$ and $\vec{n}^{\prime}$. Thus it is obvious that the coefficients $c_{A A^{\prime}}^{(1)}, c_{A A^{\prime}}^{(2)}$ and $c_{A A^{\prime}}^{(3)}$ do not depend on the information of $|\vec{n}|$, $\left|\vec{n}^{\prime}\right|$ but on the information of the relative direction between $\vec{n}$ and $\vec{n}^{\prime}$.

In particular, we note $c_{A 0}^{(1)}=-c_{A 0}^{(3)}=1$ and $c_{A 0}^{(2)}=0$ by definition. It is also helpful to note that, when $\vec{n}^{\prime} \neq \overrightarrow{0}$,

$$
c_{A A^{\prime}}^{(3)}=\left\langle\frac{1}{\tilde{\lambda}_{A^{\prime}}} \tilde{\Delta} \tilde{f}_{A^{\prime}} \tilde{h}_{a b}\right\rangle_{A}=\sqrt{a^{3}}\left\langle\frac{1}{\lambda_{A^{\prime}}} \tilde{\Delta} f_{A^{\prime}} h_{a b}\right\rangle_{A} .
$$

There is an identity

$$
c_{A A^{\prime}}^{(1)}-2 c_{A A^{\prime}}^{(2)}+c_{A A^{\prime}}^{(3)}=0,
$$

so that one among the three kinds of coefficients, say $c_{A A^{\prime}}^{(3)}$, can always be eliminated from the formulas.

\footnotetext{
20 See the beginning of $\oint 5$ for notations.
} 


\section{A.1 Scalar modes}

From the basic modes $f_{A}$ in Eq. (A1), the components of the perturbations $\tilde{\gamma}_{a b}$ in Eq.(14) can be produced.

Let us define $\tilde{\sigma}_{a b}$ as

$$
\tilde{\sigma}_{a b}^{A}:=\frac{1}{\tilde{\lambda}_{A}} \partial_{a} \partial_{b} \tilde{f}_{A}-\frac{1}{3} \tilde{\Delta} \tilde{f}_{A} \tilde{h}_{a b} .
$$

Then, $\tilde{f}_{A} \tilde{h}_{a b}$ and $\tilde{\sigma}_{a b}^{A}$ form the basis of the scalar modes of the metric perturbation $\tilde{\gamma}_{a b}$ :

$$
\tilde{\gamma}_{a b}^{(\text {scalar })}=\frac{1}{3} \sum_{A} \gamma_{A}^{(C)} \tilde{f}_{A} \tilde{h}_{a b}+\sum_{A}^{\prime} \gamma_{A}^{(S)} \tilde{\sigma}_{a b}^{A}
$$

where $\gamma_{A}^{(C)}$ and $\gamma_{A}^{(S)}$ are arbitrary expansion coefficients.

We note some relations including $\tilde{\sigma}_{a b}^{A}$,

$$
\begin{aligned}
\tilde{D}^{a} \tilde{\sigma}_{a b}^{A^{\prime}} & =-\frac{2}{3} \partial_{b} \tilde{f}_{A^{\prime}}, \quad \tilde{D}^{a} \tilde{D}^{b} \tilde{\sigma}_{a b}^{A^{\prime}}=\frac{2}{3} \tilde{\lambda}_{A^{\prime}} \tilde{f}_{A^{\prime}}, \\
\tilde{\Delta} \tilde{\sigma}_{a b}^{A^{\prime}} & =-\tilde{\lambda}_{A^{\prime}} \tilde{f}_{A^{\prime}}, \\
\left\langle\tilde{\sigma}_{a b}^{A^{\prime}}\right\rangle_{A} & =c_{A A^{\prime}}^{(2)}-\frac{1}{3} c_{A A^{\prime}}^{(3)}=\frac{1}{3}\left(c_{A A^{\prime}}^{(1)}+c_{A A^{\prime}}^{(2)}\right),
\end{aligned}
$$

where Eq. (A6) has been used in the last line of the fourth formula.

Then, it is straightforward to compute from $h_{a b}^{(\text {scalar })}:=a^{2}\left(\delta_{a b}+\tilde{\gamma}_{a b}^{(\text {scalar })}\right)$ the curvature quantities up to $O(\gamma)$,

$$
\begin{aligned}
\boldsymbol{R}_{a b}^{(\text {scalar })} & =\frac{1}{6} \sum_{A}^{\prime}\left(a^{2} \lambda_{A}\right)\left(\gamma_{A}^{(C)}+\gamma_{A}^{(S)}\right) \tilde{f}_{A} \tilde{h}_{a b}-\frac{1}{6} \sum_{A}^{\prime}\left(\gamma_{A}^{(C)}+\gamma_{A}^{(S)}\right) \partial_{a} \partial_{b} \tilde{f}_{A}, \\
\boldsymbol{R}^{(\text {scalar })} & =\frac{2}{3} \sum_{A}^{\prime} \lambda_{A}\left(\gamma_{A}^{(C)}+\gamma_{A}^{(S)}\right) \tilde{f}_{A}, \\
r_{a b}^{(\text {scalar })} & =-\frac{1}{6} \sum_{A}^{\prime}\left(a^{2} \lambda_{A}\right)\left(\gamma_{A}^{(C)}+\gamma_{A}^{(S)}\right) \tilde{\sigma}_{a b}^{A} .
\end{aligned}
$$

\section{A.2 Vector modes}

We choose the basic solutions of the eigenvalue problem, $\Delta v_{a}=-\lambda v_{a}$, with $D^{a} v_{a}=0$. We get the solution

$$
v_{a}^{(\vec{n}, \vec{\sigma}, j)}=a f_{(\vec{n}, \vec{\sigma})} \delta_{\vec{n} \cdot \vec{e}^{(j)}, 0}\left(e^{(j)}\right)_{a},
$$

where $\left(e^{(j)}\right)_{a}:=\delta_{j a}$ can be identified with the unit vector in $\mathbf{R}^{3}$ along $x^{j}$-axis $(j=1,2,3)$, and $\left\{a\left(e^{(j)}\right)^{a}\right\}_{j=1,2,3}$ forms the orthonormal bases in the $t=$ constant geometry.

$$
\int v_{a}^{(\vec{n}, \vec{\sigma}, j)} h^{a b} v_{b}^{\left(\vec{n}^{\prime}, \vec{\sigma}^{\prime}, j^{\prime}\right)}=\delta_{j, j^{\prime}} \delta_{\vec{n}, \vec{n}^{\prime}} \delta_{\vec{\sigma}, \vec{\sigma}^{\prime}}
$$

\footnotetext{
${ }^{21}$ Note that the indices are lowered by $h_{a b}$ in Eq. (12) and raised by its inverse, $h^{a b}$.
} 
From $v_{a}^{(\vec{n}, \vec{\sigma}, j)}$, we can construct the traceless tensor $\zeta_{a b}^{(\vec{n}, \vec{\sigma}, j)}=\frac{1}{\sqrt{2 \lambda_{\vec{n}}}}\left(D_{a} v_{b}+\right.$ $\left.D_{b} v_{a}\right)$, which is normalized as $\left(\zeta_{a b}^{\left(\vec{n}, \vec{\sigma}^{\prime}, j\right)}, \zeta_{c d}^{\left(\vec{n}^{\prime}, \vec{\sigma}^{\prime}, j^{\prime}\right)}\right)=\delta_{j, j^{\prime}} \delta_{\vec{n}, \vec{n}^{\prime}} \delta_{\vec{\sigma}, \vec{\sigma}^{\prime}}$.

Then, $\tilde{\zeta}_{a b}^{(A, j)}:=\sqrt{a} \zeta_{a b}^{(A, j)}$ form the basis of the vector modes of the metric perturbation $\tilde{\gamma}_{a b}$ :

$$
\tilde{\gamma}_{a b}^{(\text {vector })}=\sum_{A, j}^{\prime} \gamma_{A j}^{(V)} \tilde{\zeta}_{a b}^{A j}
$$

Wherever the detailed index structure is not essential, let us write $A$ instead of $A j$ for notational neatness, like $\gamma_{A}^{(V)}, \tilde{\zeta}_{a b}^{A}$ and $\sum_{A}^{\prime}{ }_{\gamma_{A}}^{(V)} \tilde{\zeta}_{a b}^{A}$. One can show that

$$
\left\langle\tilde{\zeta}_{a b}^{A^{\prime} j}\right\rangle_{A}=0 \text {. }
$$

The curvature quantities up to $O(\gamma)$ turn out to be

$$
\boldsymbol{R}_{a b}^{\text {(vector) }}=0 \quad, \quad \boldsymbol{R}^{\text {(vector) }}=0, r_{a b}^{\text {(vector) }}=0 .
$$

\section{A.3 Tensor modes}

We choose the basic solutions of the eigenvalue problem, $\Delta w_{a b}=-\lambda w_{a b}$, with $D^{a} w_{a b}=0, w_{a}^{a}=0$. We get the solution

$$
w_{a b}^{(\vec{n}, \vec{\sigma}, \alpha)}=a^{2} \delta_{\vec{n}_{\perp(\vec{e}(\alpha),}, \overrightarrow{0}} \delta_{\vec{\sigma}_{\perp}\left(\vec{e}^{(\alpha)}\right), \overrightarrow{0}} f_{(\vec{n}, \vec{\sigma})} \nu_{a b}^{(\alpha)}
$$

where

$$
\begin{aligned}
& \nu_{a b}^{(1)}=\frac{1}{\sqrt{2}}\left(\begin{array}{ccc}
0 & & \\
& 1 & \\
& & -1
\end{array}\right), \quad \nu_{a b}^{(2)}=\frac{1}{\sqrt{2}}\left(\begin{array}{ccc}
-1 & & \\
& 0 & \\
& & 1
\end{array}\right), \quad \nu_{a b}^{(3)}=\frac{1}{\sqrt{2}}\left(\begin{array}{ccc}
1 & & \\
& -1 & \\
& & 0
\end{array}\right), \\
& \nu_{a b}^{(I)}=\frac{1}{\sqrt{2}}\left(\begin{array}{ll} 
& 1 \\
1
\end{array}\right), \quad \nu_{a b}^{(I I)}=\frac{1}{\sqrt{2}}\left(\begin{array}{c}
1 \\
1
\end{array}\right), \quad \nu_{a b}^{(I I I)}=\frac{1}{\sqrt{2}}\left(\begin{array}{ll}
1^{1} &
\end{array}\right) \text {, }
\end{aligned}
$$

and $\vec{e}^{(1)}=\vec{e}^{(I)}=(1,0,0), \vec{e}^{(2)}=\vec{e}^{(I I)}=(0,1,0), \vec{e}^{(3)}=\vec{e}^{(I I I)}=(0,0,1)$.

They are normalized as

$$
\int w_{a c}^{(\vec{n}, \vec{\sigma}, \alpha)} h^{a b} h^{c d} w_{b d}^{\left(\vec{n}^{\prime}, \vec{\sigma}^{\prime}, \alpha^{\prime}\right)}=\delta_{\alpha, \alpha^{\prime}} \delta_{\vec{n}, \vec{n}^{\prime}} \delta_{\vec{\sigma}, \vec{\sigma}^{\prime}}
$$

Then, $\tilde{w}_{a b}^{(A, \alpha)}:=\frac{1}{\sqrt{a}} w_{a b}^{(A, \alpha)}$ form the basis of the vector modes of the metric perturbation $\tilde{\gamma}_{a b}$ :

$$
\tilde{\gamma}_{a b}^{\text {(tensor })}=\sum_{A, \alpha} \gamma_{A \alpha}^{(T)} \tilde{w}_{a b}^{A \alpha} .
$$

Let us introduce the coefficient $c_{A A^{\prime} \alpha}^{(T)}$ as

$$
c_{A A^{\prime} \alpha}^{(T)}:=\left\langle\tilde{w}_{a b}^{A^{\prime} \alpha} \tilde{\gamma}_{A} .\right.
$$


For $\alpha=i=1,2,3$,

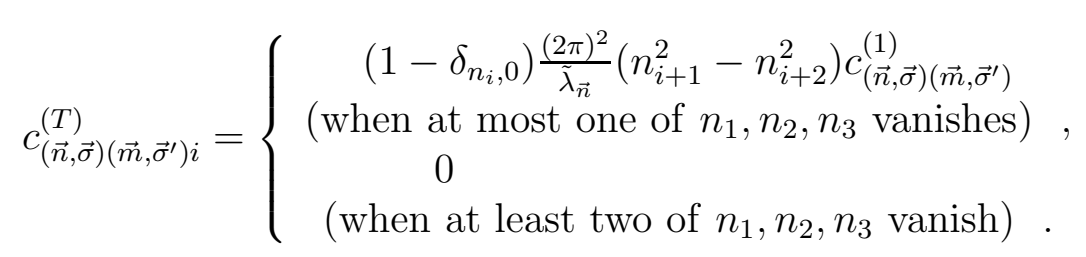

For $\alpha=I, I I, I I I$, it turns out to be $c_{A A^{\prime} \alpha}^{(T)}=0$.

The curvature quantities up to $O(\gamma)$ become

$$
\boldsymbol{R}_{a b}^{(\text {tensor })}=\frac{1}{2} \sum_{A}^{\prime} \lambda_{A} \gamma_{A}^{(T)} \tilde{w}_{a b}^{A}, \quad \boldsymbol{R}^{\text {(tensor })}=0, \quad r_{a b}^{(\text {tensor })}=\frac{1}{2} \sum_{A}^{\prime} \lambda_{A} \gamma_{A}^{(T)} \tilde{w}_{a b}^{A}
$$

where we have used a simplified index notation $A$ for $A \alpha$ for notational neatness. We follow this convention wherever the detailed index structure can be omitted, and we write simply, e.g., $\gamma_{A}^{(T)}, \tilde{w}_{a b}^{A}$ and $\sum_{A} \gamma_{A}^{(T)} \tilde{w}_{a b}^{A}$.

\section{B Basic results of the linear structure-formation theory}

We summarize the time evolution of the linear perturbations for the typical cases when (I) $\lambda_{\text {phys }} \gg c_{s} t$ and $\theta \neq 0$, (I') $\lambda_{\text {phys }} \gg c_{s} t$ and $\theta=0$ and (II) $\lambda_{\text {phys }} \ll c_{s} t$, where $\lambda_{\text {phys }}$ is the physical wave length of the perturbation mode [14].

\section{B.1 Behavior of perturbations: The case of $\lambda_{\text {phys }} \gg c_{s} t$ and $\theta \neq 0$}

A perturbation mode satisfying $\lambda_{\text {phys }} \gg c_{s} t$ does not oscillate sufficiently during the cosmological time-scale $t$. It is a long-wave length, slowly varying fluctuation compared to $t$. Let $\tau$ be the oscillation time-scale of the perturbation, $\tau=$ $\lambda_{\text {phys }} / c_{s}$. Then $t \ll \tau$, so that the only important time-scale in this situation is $t$, and perturbations naturally vary as a power of $t$.

We assume $\nu=$ constant $=c_{s}^{2}$ for simplicity.

In this case,

$$
\begin{aligned}
\delta(\vec{x}, t) & =F(\vec{x}) t^{\frac{9 \nu-1}{3(1+\nu)}}, \gamma(\vec{x}, t)=-\frac{2(1+3 \nu)}{\nu(9 \nu-1)} \delta \\
\theta(\vec{x}, t) & =\frac{(1-\nu)(6 \nu+1)}{3 \nu(1+\nu)^{2}} \frac{\delta}{t} \propto \frac{1}{\nu} t^{-\frac{2(2-3 \nu)}{3(1+\nu)}} .
\end{aligned}
$$

Here $F(\vec{x})$ is an arbitrary function determined by the initial condition imposed at a certain time $t=t_{0}$; the factor $1 / \nu$ has been shown in the last term of the last line to indicate the singular behavior for $\nu \downarrow 0$. 
As a typical situation, it is illustrative to consider the plane-wave type component of perturbation which is moving in the $x^{3}$-direction. In this case, the behavior of $\tilde{\gamma}_{33}$ and $\tilde{\gamma}_{11} \sim \tilde{\gamma}_{22}$ are

$$
\tilde{\gamma}_{33} \sim-2 \tilde{\gamma}_{11} \sim-\frac{8(6 \nu+1)}{9 \nu(1+\nu)^{2}} \frac{a^{2}}{k^{2} t^{2}} \delta \propto \frac{1}{\nu}\left(\frac{\lambda_{\text {phys }}}{c_{s} t}\right)^{2} \delta \propto \frac{1}{\nu k^{2}} t^{-\frac{1-\nu}{1+\nu}},
$$

where $k$ is the coordinate wave-number $\left(k=2 \pi a / \lambda_{\text {phys }}\right)$.

It is convenient to summarize the relation of the order of magnitude of several quantities in a symbolical manner as

$$
(|\gamma| \sim|\delta| \sim|t \theta|) \sim\left(|t \dot{\gamma}| \sim|t \dot{\delta}| \sim\left|t^{2} \dot{\theta}\right|\right) \ll\left|\tilde{\gamma}_{k l}\right| \sim\left|t \dot{\tilde{\gamma}}_{k l}\right|
$$

Regarding the rate of change of $\tilde{\gamma}_{11}$, we see that $t\left|\dot{\tilde{\gamma}}_{11}\right| \sim\left|\tilde{\gamma}_{11}\right|$. It means that $\left|\tilde{\gamma}_{k l}\right|$ decreases prominently within the cosmological time-scale. It implies that $\left|\delta\left(t_{i n}\right)\right| \sim\left(\frac{c_{s} t}{\lambda_{\text {phys }}}\right)^{2}\left|\tilde{\gamma}_{11}\left(t_{i n}\right)\right| \sim\left|\tilde{\gamma}_{11}\left(t_{i n}\right)\right| \ll\left|\tilde{\gamma}_{11}\left(t_{0}\right)\right|$ where $t_{i n}$ is the time when the perturbation of scale $\lambda_{\text {phys }}$ "enters within the horizon" due to the enlargement of the horizon scale $(\sim c t)$, viz. $\lambda_{\text {phys }} \sim c_{s} t$. It indicates the linear perturbation theory is valid even after $t=t_{\text {in }}$ 14.

\section{B.2 Behavior of perturbations: The case of $\lambda_{\text {phys }} \gg c_{s} t$ and $\theta=0$}

In this case,

$$
\delta(\vec{x}, t)=F(\vec{x}) t^{\frac{2(1+3 \nu)}{3(1+\nu)}} \quad, \quad \gamma(\vec{x}, t)=-\frac{2}{1+\nu} \delta .
$$

For the plane-wave type component of perturbation moving in the $x^{3}$-direction, the behavior of $\tilde{\gamma}_{33}$ and $\tilde{\gamma}_{11} \sim \tilde{\gamma}_{22}$ becomes

$$
\tilde{\gamma}_{33} \sim-2 \tilde{\gamma}_{11} \sim-\frac{8(9 \nu+5)}{9(1+\nu)^{3}} \frac{a^{2}}{k^{2} t^{2}} \delta \propto\left(\frac{\lambda_{\text {phys }}}{c_{s} t}\right)^{2} \delta \propto \frac{1}{k^{2}} t^{0} .
$$

On the other hand, it turns out to be

$$
t \dot{\tilde{\gamma}}_{33} \sim-2 t \dot{\tilde{\gamma}}_{11}=\frac{8 \nu}{(1+\nu)(5+3 \nu)} \delta
$$

We can summarize the relation of the order of magnitude of several quantities symbolically as

$$
(|\gamma| \sim|\delta|) \sim\left(|t \dot{\gamma}| \sim\left|t \dot{\tilde{\gamma}}_{k l}\right| \sim|t \dot{\delta}|\right) \ll\left|\tilde{\gamma}_{k l}\right|
$$

The peculiar feature of the case $\theta=0$ compared to the case $\theta \neq 0$ is that $\left|\tilde{\gamma}_{k l}\right|$ does not change prominently within the cosmological time-scale $\left(t\left|\dot{\tilde{\gamma}}_{11}\right| \sim|\delta| \ll\right.$ $\left.\left|\tilde{\gamma}_{11}\right|\right)$. 


\section{B.3 Behavior of perturbations: The case of $\lambda_{\text {phys }} \ll c_{s} t$}

A perturbation mode satisfying $\lambda_{\text {phys }} \ll c_{s} t$ oscillates sufficiently during the cosmological time-scale $t$. It is a short-wave length, fast varying fluctuation compared to $t$. Now $t \gg \tau\left(\tau=\frac{\lambda_{\text {phys }}}{c_{s}}=\frac{2 \pi a}{k c_{s}}\right.$ characterizes the oscillation period), so that we naturally pay attention to the process whose time-scale is $O(\tau)$.

In this case, various quantities oscillate with the time-scale $\tau$ :

$$
\begin{aligned}
\delta & \simeq-\gamma \simeq-\tilde{\gamma}_{33} \propto\left(\frac{\rho+p}{k c_{s} a^{4} \rho^{2}}\right)^{1 / 2} \exp -i \int^{t} \frac{k c_{s}\left(t^{\prime}\right)}{a\left(t^{\prime}\right)} d t^{\prime} \\
\theta & \simeq-\frac{1}{1+\nu} \dot{\delta} \\
|\delta| & \simeq|\gamma| \sim\left|\tilde{\gamma}_{33}\right| \sim \tau|\theta| \propto\left(\frac{\rho+p}{k c_{s} a^{4} \rho^{2}}\right)^{1 / 2} \propto \frac{1}{\sqrt{k}} a^{-\frac{1-3 \nu}{2}} \propto \frac{1}{\sqrt{k}} t^{-\frac{1-3 \nu}{3(1+\nu)}}
\end{aligned}
$$

where we have assumed $\rho \sim \rho_{\text {av }} \propto a^{-3(1+\nu)} \propto t^{-2}$.

We can summarize the relation of the order of magnitude of several quantities in a symbolical manner as (" " indicates that the difference between the bothhand sides is at most $o(\delta)$.)

$$
\begin{aligned}
(|\delta| & \left.\sim|\gamma| \sim\left|\tilde{\gamma}_{33}\right|\right) \sim(\tau|\dot{\delta}| \sim \tau|\theta|) \ll \frac{\lambda_{\text {phys }}}{c_{s} t} \ll 1 \\
\left|\tilde{\gamma}_{11}\right| & \sim\left|\tilde{\gamma}_{22}\right| \sim 0(o(\delta)), \\
(\tau|\dot{\gamma}| & \left.\sim \tau\left|\dot{\tilde{\gamma}}_{k l}\right|\right) \sim\left(\frac{\lambda_{\text {phys }}}{c_{s} t}\right)^{2}|\delta| \ll|\delta| .
\end{aligned}
$$

\section{References}

[1] For instance, G.F.R. Ellis, in "Proceedings of the Tenth International Conference on General Relativity and Gravitation", edited by B. Bertotti, F. De Felice and A. Pascolini (Reidel, Dordrecht, 1984); H. Sato, ibid.

[2] See also, A. Krasiński, Inhomogeneous Cosmological Models (Cambridge University Press, Cambridge, 1997), Chapter 8, and the references therein.

[3] M. Seriu, Phys. Rev. D53, 6902 (1996).

[4] M. Seriu, Comm. Math. Phys. 209, 393 (2000).

[5] M. Seriu, Phys. Rev. D62 \#023516 (2000).

[6] M. Seriu, Gen. Rel. Grav. 32, 1473 (2000).

[7] M. Seriu, in "Proceedings of the 8th Workshop on General Relativity and Gravitation" (K.Oohara et. al. (eds.), Niigata University, 1999), 334. 
[8] M. Seriu, Phys. Let. B319, 74 (1993).

[9] E.g., K. T. Inoue, Class. Quantum. Grav. 16, 3071 (1999).

[10] See e.g., I. Chavel, Eigenvalues in Riemannian Geometry (Academic Press, Orland, 1984).

[11] M. Kac, Am. Math. Mon. 73(4), 1 (1966).

[12] J.L. Kelly, General Topology (D. van Nostrand, Princeton, 1955).

[13] K. Yano, Metric Spaces and Topological Structures (Kyoritsu Shuppan, Tokyo, 1998), Chapters 3 and 4.

[14] For a standard treatment of the structure-formation theory, see P.J.E. Peebles, The Large-Scale Structure of the Universe (Princeton University Press, Princeton, 1980), Chapter 5. 